UCRL-CR-117755

B239746

\title{
Direct Calibration of the Yield of Nuclear Explosion
}

Keith Nakanishi

Alexi Nikolayev

June 1994

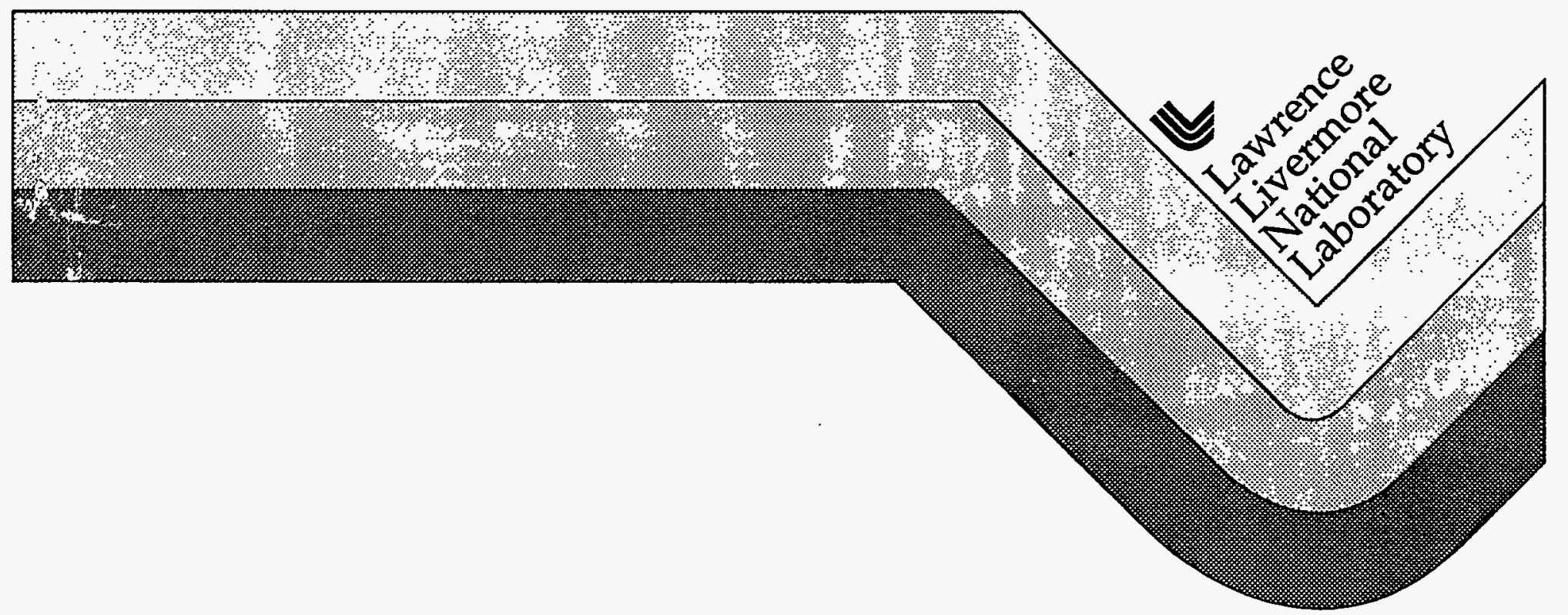

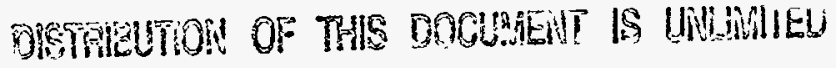




\section{DISCLAIMER}

This document was prepared as an account of work sponsored by an agency of the United States Government. Neither the United States Government not the University of California nor any of their employees, makes any warranty, express or implied, or assumes any legal liability or responsibility for the accuracy, completeness, or usefulness of any information, apparatus, product, or process disclosed, or represents that its use would not infringe privately own rights. Reference herein to any specific commercial products, process, or service by trade name, trademark, manufacturer, or otherwise, does not necessarily constitute or imply its endorsement, recommendation, or favoring by the United States Government or the University of Califomia. The views and opinions of authors expressed herein do not necessarily state or reflect those of the United States Government or the University of Califomia, and shall not be used for advertising or product endorsement purposes.

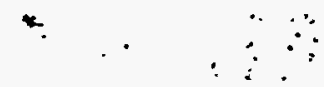




\section{DISCLAIMER}

Portions of this document may be illegible in electronic image products. Images are produced from the best available original document. 
TO University of California

Lawrence Livermore National Laboratory

Attention: Robert A. Baker, L-650

Purchase Order B239746

SYNAPSE SCIENCE CENTER/

IRIS MOSCOW DATA CENTER

OSTOZHENKA STREET, 13/12, BUILDING 1-AB,

MOSCOW, 121034, RUSSIA

$\begin{array}{lllllll}\mathbf{R} & \mathbf{E} & \mathbf{P} & \mathbf{O} & \mathbf{R} & \mathbf{T} & \text { : }\end{array}$

Direct Calibration of the Yield of Nuclear Explosion

Author: Prof. Alexei Nikolayev

LLNL Contact: Keith Nakanishi

Purchase Order Number: B239746

SYNAPSE Science Center Representative:

Mikhail Rozhkov

Telephone Number:

(095) 201.2516

Facsimile Number:

(095) 202.6934

E-Mail Number:

rozhkov@idahub.ucsd.edu

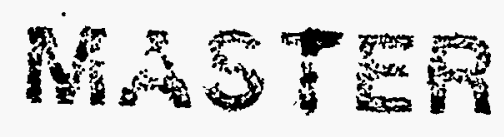


1. Introduction

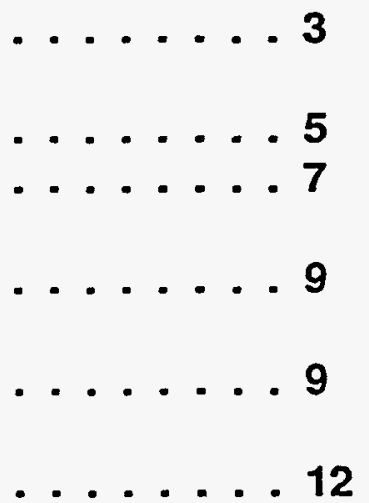

4.3. Evaluation of longitudinal wave's energy radiated by underground nuclear explosion

4.3.1. Method of UNE's yield evaluation.

Vibrational sounding of the Earth: history and state of problem.

3. Brief description of method

4. Evaluation of vibrational seismic radiation energy.

4.1. Comparison of vibro-pulse seismogram with the explosion seismogram

4.2 Vibrational monitoring of Lake Baikal rift zone

4.3.2. Evaluation of UNE seismic energy

4.4. Estimation of vibrational radiation seismic energy

4.5. Conclusion

5. Radiation of seismic waves by a vibrator

5.1. Seismic wave radiation by different types of sources

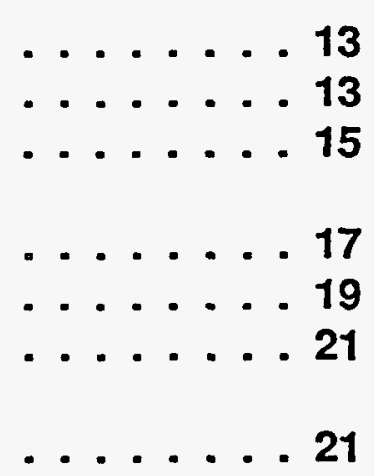

5.1.1. Radiation by force applied to an inner point of the medium and to the platform set in the mine or on the surface

5.1.2. Radiation of "twister"

5.1.3. Radiation of pulsating sphere

5.1.4. Radiation of centrifugal force circular motion

5.2. Comparison of different types of vibrators

5.2.1. Force applied to the platform

5.2.2. Vertical force applied to the surface

5.2.3. Centrifugal force. Vibrator in the shaft

5.2.4. Seismic power of vibrator for UNE calibration

5.3. Powerful seismic vibrators:

State of elaboration of the problem

5.4. Conclusion

6. Direct calibration of UNE by seismic vibrators: technique and problems

6.1. "Vibroseis" method

6.2. Frequency characteristic of the medium

6.3. Discussion, Conclusion

7. Conclusion

References

Figure captions

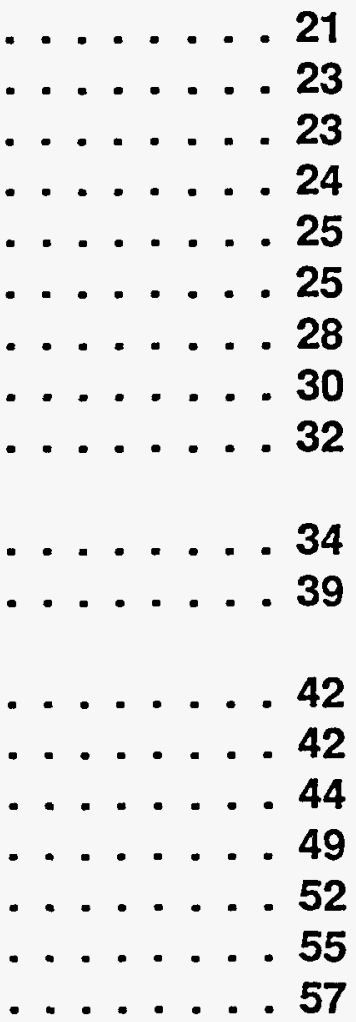




\section{Introduction}

The determination of the underground nuclear explosions (UNE) power is of great military, political and scientific importance.

The military significance of the problem is connected with the new types of nuclear weapons elaboration and with the estimation of their effectiveness.

The political significance is connected with the control of the fulfilment of UNE yield limitation agreement.

The scientific significance is bound with the solution of fundamental problems: investigation of processes attending the scattering and extinction of waves, nonlinear distortions, investigation of structure and composition of crust and mantle.

There exist several method of UNE yield determination:

- Radiochemical method;

- Nuclear and thermal radiation measurement method;

- Hydrodynamic methods;

- Seismic methods.

The first three ways use special methods and techniques for measurements in the hypocentral zone of UNE and are inapplicable for remote control. Thus, they could not be applied, for example, for the control of UNE carried out by other countries.

The seismic method allows to perform control at large distance about several thousand $\mathrm{km}$ from the UNE's epicenter, still this method is less precise than the others, that is the contact methods. The main errors in the determination of UNE's yield are due to the following causes:

- insufficiency of information about physical characteristics of shot medium;

- insufficiency of information on wave-transmission properties of the rocks between the point of detonation and the seismograph determining geometrical spreading, frictional attenuation and scattering.

To decrease the influence of these mistakes on the UNE yield there may be applied the direct calibration of yield. Seismic vibrators and chemical explosions could be used for calibration.

The application of chemical explosions has its drawbacks: insufficient energy, the necessity of calibration shot-point removal from the nuclear explosion hypocenter, difference in spectra of seismic signals radiated by nuclear and chemical explosions.

Seismic vibrators have no such shortages. A vibrator may be placed in the point where nuclear explosion is to be held, it may radiate such amount of seismic energy that will be enough for calibration of UNE and a system of seismic stations moved off at a distance of about $1000 \mathrm{~km}$. The main difficulties of the vibrator application to the calibration of UNE are of technical character. Seismic radiation power at constant force is directly proportional to square frequency and inversly proportional to wave velocity cubed. To radiate $10 \mathrm{Watt}$ 
at $1 \mathrm{~Hz}$ frequency the force more than 1000 ton is necessary. That is why the realization of the method requires the solution at least two main problems:

- evaluation of radiation energy providing the registration of seismic signal at $1000 \mathrm{~km}$ distance with signal to noise ratio not less than 10 ;

- determination of technical characteristic providing seismic calibration of UNE's yield.

The proposed way of direct UNE generated yield calibration is based on the idea of vibroseis method usage. Seismic waves are aroused by powerful vibrator in the UNE focus or epicenter. The UNE calibration is carried out by comparison of vibroseis-record with UNE seismogram.

The characteristics of seismic vibrator radiation - the form of signal and force are optional and controlled. It allows to calibrate the explosions in conventional units - in magnitudes and in yield of equivalent chemical explosion,- as well as in absolute physical units - joules of radiated seismic energy.

The present report contains the results of preliminary work on the problem. It is based upon the vibro-sounding of the Earth experience as well as the experience of the earthquakes, chemical and nuclear explosion wave field structure study.

As a result of experimental data and theoretical estimations generalization there are:

- determined the energy characteristics of seismic waves radiated by different types of vibrators;

- generalized the vibrators design principles and their technical characteristics;

- studied the methods of underground nuclear explosion (UNE) yield calibration based on the use of vibrational sounding of lithsphere;

- proposed methods of evaluation of tectonic energy released at UNE;

- worked out the recomendations for the research and development aimed at creation of method and technique of direct UNE calibration with the help of vibroseis technique;

The main result of the carried investigation is the answer "Yes" to the question if UNE calibration with the help of seismic vibrator is possible and expedient. 


\section{Vibrational sounding of the Earth: history and state of problem}

The idea of the Earth entrails investigation with the help of powerful artificial sources, vibrators, was stated by the author in 1970 in connection with the study of the Earth interior substance motion by seismic monitoring of the mantle [Nikolaev,A.V.,1970].

In 1972 A.V.Nikolaev and E.V.Artjushkov handed in to the Academy of Sciences of the USSR the proposal on detailed study of the interior of the Earth with the help of powerful seismic vibrators. This proposal was supported by the Presidium of the Academy of Sciences and there was then worked out a detailed program of investigations [Nikolaev,A.V. et al.,1974]. Within the period of 19761986 the Academy of Sciences allocated this program so it became possible to create and test models of powerful vibrators.

In 1976 on the Black Sea near Novorossijsk there was organized a 10-days workshop on the Earth vibrational sounding. Representatives of 20 different organizations presenting mainly various engineering branches participated in the workshop. The main reports of that workshop were published in 1972 as a separate collection of articles [Nikolaev,A.V.,Galkin,I.N.,1977]. Later on there was published one more collection of articles on problem of the investigation of the Earth with the help of nonexplosive seismic sources [Nikolaev A.V., Galkin,I.N.,1981].

On the first stage of the work it was planned to create and test models of vibrators based on different principles.

In the course of these researches and developments there were formed several creative groups - in Moscow, Novosibirsk, Gomel, Toljatty.

It so happened that creative groups compete with each other that either benefited and damaged the whole development of new scientific trend. The result of competition was the formation of several enlarged groups, that is the Novosibirsk, Moscow, Gomel one.

Novosibirsk engineers and geophysicists created a number of vibrators based on the principle of mass rotation with dislocation of center of gravity towards the rotation axis. The most powerful one developed a force up to 100 ton, one of such vibrators is used for Baikal Rift zone monitoring. For a number of year from 1980 up to 1990 there were held tests of several types of sources including the hydraulic vibrator constructed by academician B.V.Voitsekhovsky, Institute of Hydrodynamics, Novosibirsk. In its time, in 1980, with the help of this vibrator there was obtained a record distance of registration, that is about $2500 \mathrm{~km}$ (Novosibirsk - Zabaikalje). A vibrator developed horizontal force of about 250 ton, with $1.5-3 \mathrm{~Hz}$ frequency.

100 ton vertical vibrator usage for deep seismic sounding was hampered by its ability to function only in a stationary version, as a result it reduced the application of powerful vibrator primarily to seismic monitoring.

This also concerns 100 ton Gomel vibrator which was manufactured on technical instruction and by order of Institute of Physics of the Earth. Its 
exploitation was bound with several technical difficulties, in particular, with the problem of soil liquification. All the same, the conducted investigations of powerful seismic vibrations field allowed to find out a number of new effects and has become basic in formulation and development of a new direction - non-linear seismic. Successful usage of vibroaction upon oil deposits recovery made it possible to use the Ministry of Oil means in vibrational sources elaboration, and during 1984-1988 this financial source was the main one.

Within the period of 1989-1993 financing of investigations on vibroaction of the Earth was being constantly reduced. However, owing to the State scientifictechnical program "Global changes of environment and climate" it was possible to get small financing for scientific monitoring of the Baikal region. The work was being held by Novosibirsk geophysicists in 1991-1993.

Nowadays, the financing on this State program is substantially reduced and in 1994 works on monitoring will be stopped.

The continuation of investigations in Belorussia is also hampered. The fact is due to the Soviet Union disintegration and origination of Belorussian Republic, to introduction of new currency, to customs' complications.

The now existing result:

- There exist strong groups of engineers and scientists possessing the experience of powerful seismic vibrators creation and exploiting.

- There exist co-operation with good and stable interrelation between such institutes as:

- The Joint Institute of Physics of the Earth, Russ.Acad.Sc;

- The Computing Center and its Special Design Office of the Siberian Branch of RAS;

- The Joint Institute of Geology, Mineralogy and Geophysics of Siberian Branch of RAS;

- The Kuban's State University, Krasnodar city, Russia.;

- Scientific Research Institute of Radiophysical Measurements of High School Ministry, Niznij Novgorod, Russia;

- Special Design Bureau "Seismotechnique", Gomel, Belorussian Republic.

There are two test sites for powerful seismic vibrators investigation. One of them is in town Bystrovka area, $60 \mathrm{~km}$ South from Novosibirsk, the other is near village Uznoz, $90 \mathrm{~km}$ west from Gomel city.

Powerful stationary vibrators are preserved, groups of engineers and scientists still exist with no means for valuable work. 


\section{Brief description of method.}

The idea of the method is quite simple. Let us imagine a seismic vibrator [fig.1] fixed on vertical mine's bottom ready for the underground nuclear explosion. The vibrator exerts alternating vertical force $F(t)$ upon the mine's bottom (another orientation of force is possible), $S_{V, 0}(f)$ - Fourier spectrum of signal $F(t), f$ - frequency. This way the longitudinal and share seismic waves are created dispersing in all direction from the source $O$ and registered by seismic station $C$.

Seismic sounding is accomplished by "Vibroseis" method. In this case a vibrator radiates a long-lasting sweep - signal which is detected on a seismic station by means of the following procedure: the convolution of registered signal with the sweep-signal radiated by the source. As a result of such processing the registered seismogram is reduced to the impulse form that corresponds to a short and powerful radiation from the source.

On an impulse seismogram the form of the signal of longitudinal seismic wave arriving to the seismic station $P_{V, c}(t)$, its Fourier's spectrum $S_{V, c}(f)$.

The output spectrum and the input spectrum ratio is $S_{V, c}(f) / S_{V, o}(f)$ - this is the frequency characteristic of medium conducting longitudinal seismic wave from the source towards the seismic station. -

Now, let us take out the vibrator from the borehole and set off a nuclear device explosion in the 0 point.

The longitudinal wave $P$ originating of the explosion will be registered by seismic station in a form of a signal $P_{n, c}(t)$ with $S_{n, c}(f)$ spectrum.

The spectrum of medium being known it is possible to determine the near source longitudinal wave spectrum.

$$
S_{n, o}(t)=S_{n, c}(f) /\left(S_{V, c}(f) / S_{V, o}(f)\right) \text {. }
$$

This is the sought quantity function characteristic of radiation energy.

The UNE yield may be calibrated by this function, it is also possible to calibrate UNE by the energy of radiated longitudinal waves or by some other quantities determined with the help of function $S_{n, 0}(f)$.

The described method could be modified in different ways.

In particular, the calibration may be performed using the principle of reciprocity and sounding the seismic ray track in reverse direction. To do it, it is necessary to establish a seismic vibrator in point $C$ and a seismograph in a borehole.

The account of seismic radiation directivity diagram, the power of vibrator's radiation, discrepancy of sounding vibrosignal spectrum to that of signal aroused 
by nuclear explosion, detection of vibrosignal upon microseisms background in the registration point, the account of tectonic energy released at the explosion, methods of underground nuclear explosion yield calibration, - all these aspects are prominent and they need special additional observations and investigations to be solved.

A number of complications is bound with "Vibroseis" technique application. All these problems also need separate consideration and further special investigations. 


\section{Evaluation of vibrational seismic radiation energy}

Evaluation of vibrational seismic energy is necessary for the determination of the vibrator technical characteristics, the main of which are pick force and frequency band. These characteristics determine radiation power, the necessary duration of vibrosignal, the possibility of its frequency spectrum forming.

The amplitude of registered vibro-pulse signal depends on the power and duration of sweep-signal generated by source. That is why the similar effect could be got by different power and duration of radiation. To eliminate such uncertainty in our evaluations let us fix the duration of radiated signal and consider it equal to one hour.

Let us also settle seismic energy radiated by vibrator. Let us think that energy radiated as longitudinal $P$-waves is equal to energy radiated in the same $P$ wave by underground nuclear explosion equal to one kiloton (kt): An underground nuclear explosion radiates broad-spectral band signal and a considerable part of seismic energy is held in high-frequency oscillations absorbed and scattered at comparatively small distances of wave path. So in our estimations we shall consider only that part of underground nuclear explosion seismic energy which is held in seismic signal's frequencies band registered at more than $100 \mathrm{~km}$ distances from the source.

The vibrator's seismic yield evaluation may be done in two ways.

The first way is the comparison of vibro-pulse seismogram with chemical explosion seismogram and reevaluation of obtained result in respect to an explosion of one kiloton yield and duration of vibrators work of one hour.

The second way is the calculation of $P$-wave seismic energy radiated by underground nuclear explosion. For this purpose it is necessary to use data on wave $P$ registered on the surface amplitude and prevailing period as well as quality factor of longitudinal wave in crust and upper mantle.

\subsection{Comparison of vibro-pulse seismogram with the explosion seismogram.}

Unfortunately, the problem of vibrator calibration by explosion was practically paid no attention. There is only one example known to us which was obtained in the Institute of Geophysics of Siberian Branch of Russian Academy of Sciences with the help of 100 -ton force stationary seismic vibrator. The vibrator's construction is traditional. Two rotating masses are established on one platform. They are set in motion by electric engine and rotate in opposite directions in such a way that force horizontal component is compensated and the vertical one is summarized. The vibrator is set not far from Novosibirsk. The soil is $5-$ meter sandy loam layer disposed on an argillaceous schists.

On fig.2a it is. shown a vibro-pulse seismogram. The vibrator radiated continuous sweep-signal of $6-9 \mathrm{~Hz} 40$ minutes in duration. Pick force changed from 35 to 80 ton during it. Vibrational signal was registered at $300 \mathrm{~km}$ distance 
from the source by single vertical seismic receivers fixed on short profile. The profile is orientated towards the direction of source-receiver with distance between seismometers $100 \mathrm{~m}$.

On fig. $2 b$ it is shown the seismogram of 4 ton TNT quarry explosion. The explosion is held in limestone, the charge being installed so as to loosen the rock. The distance from the quarry up to registration point was $250 \mathrm{~km}$. The explosion was registered by the same instruments as vibrator. Time interval between the very moment of explosion and the starting time of vibrator was about one hour that is why the microseismic backgrounds intensity is practically the same on both seismograms.

Comparison of records shows that the intensities of two main groups of volume waves of wave $P n$ refracted below the Earth crust and reflected from Mocho boundary $P^{M}$ wave are a bit different. On the explosion seismogram the $P n$ - wave has a 1.5.time less pick, its dominant frequency is somewhat higher and is equal to $8.0 \mathrm{~Hz}$ whenever the prevailing frequency of $P^{M}$ wave is equal to $7.1 \mathrm{~Hz}$.

At the same time $P^{M}$ wave on a vibro-pulse seismogram has $1.5-2.0$ times higher pick that on an explosion seismogram. The waves $P n$ and $P^{M}$ prevailing frequencies on each seismogram are practically the same. The identification of $P^{M}$ wave with Mocho reflection is made by DSS-experts.

Naturally, our comparison of seismograms is not quite proper as they correspond to different sources type, positions and different epicentral distances. Taking it all in account it is no sense in a more detailed comparison of seismograms and one may satisfy with just an approximate evaluation.

For such evaluation one may take the intensity of both $P n$ and $P^{M}$ wave to be equal. Thus we arrive at the conclusion of both vibrator and explosion aroused longitudinal waves energy equality. For further evaluations let us consider that in both experiments wave registration was carried out at $300 \mathrm{~km}$ distance from the source and that pick force of vibrational source is $100-$ ton, the duration of sweep-signal is 1 hour and not 40 minutes. All these suppositions lead to a somewhat underestimated evaluation of vibrational source energy in ratio to an explosion.

To estimate the amount of mean seismic energy which corresponds to $1 \mathrm{kt}$ explosion we shall use the following formulae to the charge of explosion $W \mathrm{kt}$, $P$-wave magnitude $m$ and source energy $E$ Joule [Kiritchenko et al., 1993]:

$$
\begin{aligned}
& \lg E=4.8+1.5 m \\
& m=4.4+0.8 \lg W \\
& \lg E=11.4+1.2 \lg W
\end{aligned}
$$

The coefficients depend on the depth of the source, near-source geology, and may be changed according to specific conditions.

The charge difference between $1 \mathrm{kt}$ and 4 ton is 250 times, this corresponds to 754 times of energy difference. 
Thus, the 100ton vibrator should work 754 hours to generate the seismic energy which equals to $1 \mathrm{kt}$ explosion. The question may be put in another way: what force should be created by vibrator to produce within one hour the same seismic energy as an explosion of $1 \mathrm{kt}$ ? Seismic radiation power is in proportion to a square force, so the vibrator would have an effect of 2700 - ton force, that is to say its seismic effect would be equal to $1 \mathrm{kt}$ explosion.

Our evaluations do not consider that $1 \mathrm{kt}$ yield nuclear explosion has a lower frequencies spectrum than 4ton TNT explosion, so the obtained result should be treated as rough estimation.

The investigation of seismic power given back by this vibrator to longitudinal, transversal and surface Raleigh waves showed that radiation frequency characteristic has its maximum at $4.5 \mathrm{~Hz}$ frequency making up 1500 Watt and in frequency band of $6-9 \mathrm{~Hz}$ mean radiation yield is 1200 Watt. It is known that radiation power into longitudinal wave by a vertical vibrator makes $7 \%$ from total power, which is about 85 Watt in our case, and radiation energy is $2.0 \cdot 10^{5}$ Joule. This value may be accepted as a quarry explosion seismic energy.

Thus we have calibrated the vibro source by quarry explosion and found out that its work during 1 hour is equal to 4 ton quarry explosion in its seismic effect, we have calibrated the quarry explosion by vibrosource and found that its seismic energy radiated into $P$ wave is about $2.0 \cdot 10^{5}$ Joule.

According to the classification of explosions such energy corresponds to magnitude of

$$
m=\frac{\lg E-4.8}{1.5}=\frac{\lg \left(2.0 \cdot 10^{5}\right)-4.8}{1.5}=0.3 \text {. }
$$

This is the rough estimation of the magnitude of 4 ton explosion calibrated by seismic vibrator. At the same time we can get the magnitude of this explosion using the formula

$$
m=4.4+0.8 \lg W=2.5 \text {. }
$$

The two orders of difference of the magnitude obtained by different ways is too large to explain it by conditions of explosion and local geology. Unfortunately, the seismogram on fig. 2 is registered by uncalibrated tools and so we are not able to define the quarry explosion magnitude. The obtained evaluation $m=0.3$ is obviously underestimated. However, now the experience of non-explosive sources usage - that is vibrators and powerful airguns give grounds to think that the existing seismic sources power scales are displaced. Seismic energy corresponding to certain source magnitude turns out to be less than the estimations given by formulae of magnitude to energy transfer. 


\subsection{Vibrational monitoring of Lake Baikal Rift zone.}

Experience of the Baikal Rift zone vibrational monitoring corroborates our suppositions of magnitude and power scale displacement.

The works were carried out by the Institute of Geology and Geophysics of Siberian Branch of Russian Academy of Sciences in 1991 - 1993 [Yushin et al., 1994].

The field experiment was organized in a following way. Seismic vibrator was situated on the east shore of Lake Baikal near village Kharaz as it is shown on fig.3. The distance between vibrator and the station is $125 \mathrm{~km}$.

In these works there was used a vibrator of the described type. There were used sweep-signals of $5.5-9.5 \mathrm{~Hz}$ with non-linear frequency changes, the duration was 20 minutes. A vibrator was fixed on a 5-meter layer of sand clay upon a thick pebble layer. Mean radiation power in a frequency range of $5.0-9.5 \mathrm{~Hz}$ about 1300 Watt, $P$-wave power is 90 Watt.

Portable 12-channel seismic station registered and processed the vibrosignals. Vertical seismometers were established on a short section orientated towards the source. The distance between seismometers was $100 \mathrm{~m}$.

The aim of the investigation was systematic seismic radiation performed to reveal seismogram changes bound with temporal variations of seismic waves velocities distribution in lithosphere.

That was the reason why seismic radiation faced demands of high signal to noise ratio and 20 -minutes sweep-signals were many times repeated.

On fig.4a-d there are shown four seismograms corresponding to 20 -minutes actions. These seismograms illustrate repeatability of the source. They are characterized by relatively small records picks in ratio to seismic noises level. The first diffracted wave arriving in $21.5 \mathrm{sec}$ is hardly seen on the record. Most distinctly seen is a group of short $P g$ waves and three(!) groups of waves $23.5-25 \mathrm{sec}$ refracted at Mocho discontinuity. Such wave identification is given by Novosibirsk geophysicists. On fig.4e seismogram is shown corresponding to 24 individual actions, total duration of radiation is 8 hours. This seismogram is presented with variable magnification along the record. Its initial part up to 22 $\mathrm{sec}$ has 6 time magnification, at $22-23 \mathrm{sec}$ interval - magnification 8 times decreased and further remains constant.

Unfortunately, as it was in the previous experiment registration channels did not have absolute calibration. That is why the earthquakes energy class (which is equal to $\lg E$ ) estimation corresponding to vibro-pulse seismogram was performed on the base of experts' opinions. The seismogram of 8 -hour work of vibrator with given epicentral distance of $125 \mathrm{~km}$ correspond to an earthquake of 6.5 energy class, $m=1.1$ which is in good accordance with the previous estimation obtained at 1 -hour work of vibrator fixed in Novosibirsk area. Difference of magnitude corresponds to approximately 5 -time difference in energy radiation. In this particular case the duration of radiation increases 12 
times in comparison with Novosibirsk tests and radiation energy also increases 12 times, so one might expect that equivalent energy 6.2 class will be not 6.5 but 6.2. The obtained difference seems to be due to two circumstances: the first is the peculiarities of lithospheric structures and seismic waves propagation paths. The second is an error of experts' estimation.

One should specially mark good identity of $a, b, c, d$ seismograms. The question of identify of powerful seismic vibrators work was specially studied by Novosibirsk geophysicists. It was stated that pick identity of vibrational seismograms is not less than $1 \%$ and phase identity is not less than 0.001 second.

\subsection{Evaluation of longitudinal wave's energy radiated by underground nuclear explosion}

For estimation of UNE's energy radiated in seismic waves there might be, in principle, used different types of waves: - longitudinal $P g$ and $P n$, transversal $S n$, wave of surface type $L g$ spreading in the Earth crust as well as seismogram's "tail" - coda.

Transversal wave $S n$ forms in consequence of medium lateral inhomogeneity existence near the source distorting radiation symmetry. That is why it seems to be senseless to evaluate the energy of explosion by the wave it should not radiate. The same could be said about $L g$.

Our estimation on base of $P n$ wave is expedient. Seismic wave $P n$ is a longitudinal wave refracted into the very upper part of mantle which interfere with multiple refracted waves spreading under Mocho discontinuity.

We should note that our estimation concerns only that part of seismic energy which is in frequency band of the registered signal.

\subsubsection{Method of UNE's yield evaluation}

Let us imagine that the medium is horizontally homogeneous and the seismic wave velocity $V$ smoothly grows with the depth $z$. In such case seismic rays would be the curves lying in vertical plane

$$
V^{*}=\frac{R}{r} \cdot V(r)
$$

could be applied; where $V^{*}$ - is an apparent velocity on the Earth surface; $R=6370 \mathrm{~km}$ - the Earth's radius, $r$ - the distance between the ray's lower point and the Earth's center, $V(r)$ - seismic wave velocity in the ray's lower point.

With the epicentral distance of $1000 \mathrm{~km}$ the depth of seismic ray penetration $R-r$ is equal to about $200 \mathrm{~km}$, so the $r / R \cong 0.97$ value we should accept to be equal to one, that is to say we could consider the plane approach. 
Let the seismic source be situated in point $O$ and radiate a short signal with duration $\tau$, prevailing period $T$.

$O C_{1}, O C_{2}$ - seismic rays, $i_{1}$ and $i_{2}$ the exit angles in $C_{1}$ and $C_{2}$ points. As the depth of the source is small, the ray's outlet angles in point 0 are also equal to $i_{1}$ and $i_{2}$ (fig.5).

Let us mark the vertical component of medium's particle velocity pick on the Earth surface as $\stackrel{\circ}{U}$ If $\stackrel{\circ}{U}$ value between $C_{1}$ and $C_{2}$ points changes insignificantly, than it may be taken constant and equal to the mean value in epicentral distances interval $\Delta_{1} \Delta_{2}$.

Seismic energy radiated into angle of solid formed by rays from the sourcecoming to $C_{1}$ and $C_{2}$ points rotation around vertical axis, may be determined by kinetic energy of medium's subsurface circular layer oscillations. Inner and outer rings radii $\Delta_{1}$ and $\Delta_{2}$, depth $h$ are defined by signal $\tau$ duration, velocity $V$ and mean meaning of angle of arrival $i$ in the interval $\Delta_{1} \Delta_{2}$ :

$h=V \cdot \tau \cdot \cos i$

Mean kinetic energy of ring's oscillations, connected with arriving $P$ wave.

$$
E\left(\Delta_{1}, \Delta_{2}\right)=m \cdot(\stackrel{\circ}{* 2} / 2) / 2
$$

Where $\left(\stackrel{\circ}{U^{* 2}} / 2\right)$ - mean value of squared particle velocity of arriving $P$ wave, $m$ - mass of ring's volume:

$$
m=2 \pi \cdot\left(\Delta_{2}^{2}-\Delta_{1}^{2}\right) \cdot h \cdot \rho
$$

$\rho$ - density.

Value $U^{\circ}$ is connected with vertical component of particle velocity on surface $\dot{U}$ with "coefficient of conversion", which characterises the reflection and conversion of arriving $P$-wave into reflecting share wave:

$$
\stackrel{\circ}{U}^{*}=k \stackrel{\circ}{U}, \stackrel{\circ}{U}=k^{-1} \dot{U}^{*}
$$

Therefore we get the following approximate formula for $P$ wave seismic energy arriving to the Earth's surface inside the ring:

$$
E\left(\Delta_{1}, \Delta_{2}\right)=2 \pi \cdot\left(\Delta_{2}^{2}-\Delta_{1}^{2}\right) \cdot V \cdot \tau \cdot \rho \cdot \cos i \cdot k^{-2} \cdot\left(\stackrel{\circ}{U^{2}} / 2\right) / 2
$$

This is the part of seismic energy which has reached the Earth's surface after absorption and Mocho discontinuity passing loss. Absorption losses were evaluated by exponent : 


$$
\exp (-\pi t / Q T)
$$

$t$ - time of wave propagation from the source towards the registration point, $T$ the prevailing period of signal , $Q$ - quality factor considered to be constant along the seismic ray. Losses on Mocho discontinuity passing from crust to mantle and from mantle to crust are reduced to $\bar{M}$ coefficient which is calculated for flat boundary and velocity ratio of longitudinal and transversal waves $\sqrt{3}$.

Energy arriving to the surface within the ring is radiated by source into the angle of solid

$$
2 \pi \cdot \sin i \cdot \sin \left(i_{2}-i_{1}\right)
$$

where $i_{1}$ and $i_{2}$ - angles of seismic rays arriving to $C_{1}$ and $C_{2}$ points, $i=\left(i_{1}+i_{2}\right) / 2$ - mean angle. This approximate formula is true when $i_{2}-i_{1}$ is a small angle. If radiation of the source is isotropic then its total radiation into space is

$$
4 \pi /\left(2 \pi \cdot \sin i \cdot \sin \left(i_{2}-i_{1}\right)\right)
$$

times more than into considered angle of solid. Now taking into account that passing the Mocho discontinnity twice the wave energy is reducing $\bar{M}$ times, we can write the whole expression for seismic energy radiation

$$
\begin{aligned}
E & =\frac{E\left(\Delta_{1}, \Delta_{2}\right) \cdot \exp (\pi t / Q T) \cdot \bar{M}}{\sin (i) \cdot \sin \left(i_{2}-i_{1}\right)}= \\
& =\frac{\pi\left(\Delta_{2}^{2}-\Delta_{1}^{2}\right) \cdot V \cdot \tau \cdot \rho \cdot \cos i \cdot k^{-2} \cdot \stackrel{U}{ }^{2} \cdot \exp (\pi t / Q T) \cdot \bar{M}}{2 \sin i \cdot \sin \left(i_{2}-i_{1}\right)}
\end{aligned}
$$

This expression is obtained with a great number of assumptions. It might be modified when applied to a more common cases, for example, when signal's spectrum has a complicated form, $Q$ is dependent on depth and changes by lateral, radiation is not isotropic etc. We confine.ourselves to this simple formula because we mean to make only a rough estimation of energy for strictly schematic and averaged parameters of medium and wave field.

\subsubsection{Evaluation of UNE seismic energy}

Among the variety of observed patterns of $P n$ wave corresponding to nuclear explosion in different conditions the data on explosions on Semipalatinsk test site are of the greatest interest. Similar in character change of amplitude and prevailing $P n$ wave period was observed for explosions held in the north Caucasus, north by- Caspian and in city Perm area. All these are brought together and are presented on fig. $6 \mathrm{a}$ in a form of dependence of amplitude on 
particle velocity and $P n$ - wave prevailing frequency from epicentral distance $\Delta$ led to an explosion of $1.0 \mathrm{kt}$ yield. The width of shading corresponds to standard scattering of individual values.

Fig. $6 \mathrm{~b}$ shows the diagram of the wave prevailing period changes with epicentral distance. Shading shows standard scattering of values.

These diagrams were based upon observation data of $P n$ - wave on seismic stations situated in south Turkmenia, Uzbekistan, Khazahstan and Kirgizia. The majority of stations are situated on crystalline rocks.

As it is seen on the diagram of the particle velocity amplitude change for $1 \mathrm{kt}$ UNE shown on the Fig.6, the diagram cools with distance. It is expedient to estimate the UNE seismic energy by the part of diagram within the range of $500-1000 \mathrm{~km}$ distances, where it is characterized by linear logarithmic decrease.

To apply formula defining explosion energy there are given values forming it. For this purpose it is necessary first of all to set the wave velocity model of the Earth crust and the uppermost part of mantle. Let us take the most simple and typical model: homogeneous crust and linear increase of velocity due to depth in the uppermost mantle. Longitudinal wave velocity in the Earth crust is

$6.0 \mathrm{~km} / \mathrm{sec}$, crust's thickness is $40 \mathrm{~km}$, velocity under Mocho discontinuity is $8 \mathrm{~km} / \mathrm{sec}$, velocity gradient $0.008 \mathrm{sec}^{-1}$.

This ideal section is in accordance with DSS data on a number of regions of Central Asia, in particular, on the Central Kazakhstan: (Kopet-Dugh - the Aral Sea cross section, the Turan plate) [Ryaboy,V.Z.,1979] (Fig.7).

In other specific cases there might be used other models, the advantages of our model are in its simplicity, in its being noncontradictory to the notions on lithosphere structure in Semipalatinsk test site region.

Ray approach was used to determine $\bar{M}$ coefficient. With angle $i$ near to angle of total refraction, the ray approach does not give quite correct solution for $\bar{M}$ coefficient determination, though in our case with the epicentral distance of more than $500 \mathrm{~km}$ they would make not more than $10 \%$ and so the ray approach may be accepted.

The lithosphere quality factor we may take equal to 200 . This is deliberately low meaning and we take it to get the upper estimation of energy value.

Let us take the Earth crust density $\rho$ equal to $2600 \mathrm{~kg} / \mathrm{m}^{3}$, the wave prevailing period $T=0.6 \mathrm{sec}$, duration of signal $\tau=2 \mathrm{sec}$.

Several estimation of $E$ value may be done by $100 \mathrm{~km}$ intervals of epicentral distances:

$500-600 \mathrm{~km}, 600-700 \mathrm{~km}, 700-800 \mathrm{~km}, 800-900 \mathrm{~km}, 900-1000 \mathrm{~km}$

For the small epicentral distances the conditions of ray theory are worse implemented, the angle of departure being too near to the angle of total refraction. 100 - kilometer intervals answer the condition of relatively small change of $t$ and $i$ values. 
$\stackrel{\circ}{U}$ values are taken from the diagram on fig. 6 for UNE of $1 \mathrm{kt}$ yield. Table 1 shows the values of main parameters being a part of formula for seismic radiation energy and the energy itself for five epicentral distances intervals.

\section{Table 1}

\begin{tabular}{|c|c|c|c|c|c|}
\hline$\Delta, \mathrm{km}$ & $500-600$ & $600-700$ & $700-800$ & $800-900$ & $900-1000$ \\
\hline$\stackrel{\circ}{U}, 10^{-7} \mathrm{~m} / \mathrm{sec}$ & 15 & 9 & 5.5 & 3.3 & 2.0 \\
\hline$K^{-2}$ & 0.56 & 0.58 & 0.52 & 0.50 & 0.48 \\
\hline $\exp (\pi t / Q T)$ & 6.6 & 9.2 & 13.0 & 18.3 & 25.5 \\
\hline $\bar{M}$ & 1.65 & 1.40 & 1.30 & 1.25 & 1.20 \\
\hline$E, 10^{9} \mathrm{Joule}$ & 27.2 & 13.9 & 6.42 & 4.45 & 2.63 \\
\hline
\end{tabular}

As it is seen, the estimations of UNE energy of $1 \mathrm{kt}$ yield turned out to be significantly different, the scattering being about one order. It is usually believed that seismic energy of UNE of such yield is about $2.5 \cdot 10^{11}$ Joule, the estimations obtained by us, though near, are distinctly less.

It is remarkable that the obtained estimations of $1 \mathrm{kt}$ UNE energy are decreasing with epicentral distance. This could be connected with different things. One of the main ones is that the quality factor depends on the depth and it is not so low in the uppermost mantle. The second thing is connected with applicability of the ray theory. Consistency of results increases with distance, so the estimations obtained for the range of $700-1000 \mathrm{~km}$ could be considered as more reliable. Taking this into account we should accept the value $5 \cdot 10^{9} \mathrm{Joule}$ as the amount of $P$-wave energy radiated by $1 \mathrm{kt}$ UNE into frequency band $1-2.5 \mathrm{~Hz}$.

\subsection{Estimation of vibrational radiation seismic energy.}

The fore-cited estimations of energy concern explosions of different yield and different frequency bands. To compare these results let us recalculate. the obtained estimations of $1 \mathrm{kt}$ yield.

A 4 ton TNT explosion radiated energy of $2.0 \cdot 10^{5}$ Joule into longitudinal wave. We might indirectly evaluate energy and magnitude of $1 \mathrm{kt}$ explosion using this result. 
It is considered that at 250 times increase of the yield from 4 ton to $1 \mathrm{kt}$ should correspond to 754 times energy increase. This gives evaluation of $1 \mathrm{kt}$ explosion energy

$$
2.0 \cdot 10^{5} \cdot 754=1.5 \cdot 10^{8} \text { Joule. }
$$

That is significantly less than $2.5 \cdot 10^{11}$ Joule value which is accepted as $1 \mathrm{kt}$ energy for Semipalatinsk test site.

The experience of vibrational seismic sounding in Lake Baikal region showed that the eight hours work of a vibrator corresponds to an earthquake of 6.5 energy class, according to expert evaluations the radiated energy is equal to $3.2 \cdot 10^{6}$ Joule.

This is in accordance with $4.0 \cdot 10^{5}$ Joule evaluated in one hour. Seismic power of Baikal vibrator of about 90 Watt is close to Novosibirsk vibrator power of 85 Watt. The difference in values is negligibly small in spite of low accuracy of our estimations, different conditions of radiation and wave propagation.

Thus, experimental estimation based on vibro-pulse seismograms comparison with explosion and earthquake records are in satisfactory accord in between and in a sharp discrepancy with an accepted value of seismic energy of $1 \mathrm{kt}$ nuclear yield. This part of investigations is performed on data about relatively short period seismic waves, $5-10 \mathrm{~Hz}$.

For waves characterized by $0.6 \mathrm{sec}$ prevailing period which corresponds to $1 \mathrm{kt}$ nuclear explosion and several hundred kilometers distance there were obtained a more close $1 \mathrm{kt}$ UNE energy estimations though they were $1-2$ period lower: $10^{9}-10^{10}$ Joule in ratio to $2.5 \cdot 10^{11} \mathrm{Joule}$. For further evaluations let us take $1.5 \cdot 10^{8}$ Joule for frequency band $5-10 \mathrm{~Hz}$ and $5.0 \cdot 10^{9}$ Joule for $1-2.5 \mathrm{~Hz}$ range as standard. The discrepancy of obtained evaluations leaves us a somewhat bewildering and discontented feeling. It's quite evident that the immediate task of scientific researches with vibrational sources should become the study of energy - magnitude - seismic waves intensity ratio in regional and teleseismic zone.

The result of such research might become the revision of our notions upon yield radiation energy of seismic explosions and upon dissipation characteristics of lithosphere.

To radiate seismic energy of $1.5 \cdot 10^{8}$ Joule in an hour a vibrator must radiate seismic wave of $4.1 \cdot 10^{4}$ Watt power at $5-10 \mathrm{~Hz}$ frequency and to radiate $5.0 \cdot 10^{9}$ Joule it must radiate a wave of $1.4 \cdot 10^{6}$ Watt power at $1-2.5 \mathrm{~Hz}$ frequency. The question of technical means of such source creation is looked upon in the next part, but even without special investigation it is possible to say that the creation of such powerful low-frequency vibrator is actually impracticable problem.

Numbers cited for low-frequency radiation are, probably, "pessimistic estimation" and should be looked upon as an upper limit as yet on base of which 
it is possible to plan some measure aimed at the main difficulty overcoming - the necessity to radiate large portions of energy into relatively long period seismic waves.

For calibration of small yield explosions the use of high frequencies, up to $40 \mathrm{~Hz}$ is of special interest. Nowadays we have no data on UNE's high frequency radiation characteristics and the question of applying high frequencies for UNE's calibration with the help of vibrator needs special study. Anyhow, the creation of high frequency vibrator of large yield is a less complicated technical problem than that of a powerful low frequency vibrator. The factor might become decisive in a choice of seismic signals calibration parameters.

\subsection{Conclusion}

Estimations of calibrated signal's energy orientated at UNE of $1 \mathrm{kt}$ yield are made on the base of different approaches:- comparison of 4 ton explosion's seismogram and 100 ton vibro-pulse seismogram of Novosibirsk vibrator;- expert evaluation of Baikal vibrator seismogram in terms of equivalent local earthquake class (according to adopted in the former USSR scale of the earthquake power classification the class is equivalent to a logarithm of seismic energy reduced to certain standard conditions);- calculation of seismic waves energy on the base of experimental data on surface dislocation velocity changes for $\mathrm{Pn}$ wave for UNE on the Semipalatinsk test site;- evaluation of UNE energy on the base of energy empiric dependence upon yield for the Semipalatinsk test site.

The obtained results are of different degree of certainty and reflect only vagueness of our knowledge that should be overcome by study of power characteristics of waves radiated by UNE, chemical explosions, vibrators.

1. Seismic energy radiated by $1 \mathrm{kt}$ UNE in $1-2.5 \mathrm{~Hz}$ frequency band evaluated on the basis of experimental data on Pn wave intensity change at 500$1000 \mathrm{~km}$ distance from the source is at least two orders less than evaluation given by empiric formula which bind energy and UNE's yield.

2. $1 \mathrm{kt}$ UNE seismic energy estimated on the basis of 4 ton explosion registration calibrated by a vibrator, is at least an order less than the value got on the basis of $P n$ wave intensity data. The mentioned differences are due to imperfection of the applied method of evaluation based on simplified assumptions as well as to various conditions of seismic waves generation and spreading from the source towards registration points. Nevertheless, these differences are too large to be explained altogether by these reasons. If it is so, we should assume that the existing empiric evaluations of UNE's and chemical explosions energy are systematically increased.

3. The obtained evaluations of seismic energy amount equivalent to $1 \mathrm{kt}$ UNE in $1-2.5 \mathrm{~Hz}$ and $5-10 \mathrm{~Hz}$ frequency band are different, to a great extent this may be due to the fact that empiric regularity binding an explosion yield and radiated seismic energy do not describe well enough the correlation between these charateristics for small yield explosion of about few tons. In such case the 
calibration of UNE of $1 \mathrm{kt}$ or greater yield by small yield chemical explosions do not give quite probable result, first of all, due to the difference in large and small yield explosions spectral composition. Seismic vibrator, though having very small power of radiation could imitate frequency characteristic of powerful UNE. Here lies its principle difference and advantage over calibration chemical explosion.

4. The chosen calibration energy threshold of $1 \mathrm{kt}$ is conditional. Bearing in mind that the aim of calibration is the determination of medium frequency characteristic corresponding to a given disposition of source and registration point, the seismic vibrator radiation energy should be estimated upon the vibrosignal registration conditions with certain exceeding over noise level. Such estimation is made in the next part of the report.

5. The obtained experience of vibrator radiated seismic waves shows that in many cases positive result was obtained counter to preliminary calculations which summarized the experience of the earthquakes and UNE study. At the end of 70-s when comparatively large costs were spent on Novosibirsk vibrator design but the problem of precise managing was not yet solved, the participants of "Vibrational sounding of the Earth" program had rather hard times. Academician G.I.Marchuk who was then the vice-president of the Academy of Sciences and the head of Siberian Branch of RAS set a condition:" to prove a theorem of existence", that is, to find a signal from a vibrator at more than 1000 $\mathrm{km}$ distance. The task seemed impossible even to ourselves, geophysicists and engineers. To our mutual surprise, the harmonic signal was safely detected by spectral analyses method at $1100 \mathrm{~km}$ distance from Novosibirsk, in Central Kazakhstan. This saved the program. In vibrational sounding of the Earth, Nature takes the part of experimenter. 


\section{Radiation of seismic waves by a vibrator}

Let us consider the question of seismic waves radiation for two cases with a vibrator installed in a mine and on the Earth's surface. There are different possible construction of vibrators, different types of action. For instance, those used in seismic prospecting create vertical or horizontal force applied to the surface. To be set in mines and tunnels there could be used not only these types but the vibrators which create circulating force and the vibrators which do not posses the inertial mass but thrust asunder the opposite walls. The theory of these sources is stated in a monograph [Isakovich,1973].

\subsection{Seismic wave radiation by different types of sources}

Let us consider the following types of sources

- force applied to an inner point of the medium and to the Earth surface;

- source creating the turning moment("twister");

- pulsating sphere;

- centrifugal force moving circularly.

\subsubsection{Radiation by force applied to an inner point of the medium and to the Earth surface}

The harmonic force acting upon the small dimension (compared to wave length) mine's bottom radiates as much force applied to a point in an unlimited homogeneous medium. In this case the seismic radiated power [Isakovich,M.A. 1973]:

$$
J(f)=\frac{\pi f}{6 p}|F(f)|^{2} \cdot\left(\frac{2}{V_{s}^{3}}+\frac{1}{V_{p}^{3}}\right)
$$

where $F(f)$ - the amplitude of force upon the frequency $f$ of radiation, $\rho$ - the medium density, $V_{p}$ and $V_{s}$ - velocities of longitudinal and transversal waves. The first item corresponds to radiation of transversal wave, the second - of longitudinal one. In particular, with equal Lame coefficients $(\lambda=\mu) \quad V_{p}=V_{s} \sqrt{3}$ and (1) comes to:

$$
J(f)=\frac{\pi f^{2}}{3 \rho V_{s}^{3}}|F(f)|^{2} \cdot(1+1 / 6 \sqrt{3})=0.091 \frac{4 \pi f^{2}}{\rho V_{s}^{3}} \cdot|F(f)|^{2} .
$$

In comparison with it we'll state that the oscillating force radiation yield when applied along the normal towards the surface of solid semispace makes [Gustchin V.V. et.al., 1981] with $\lambda=\mu$ 


$$
J_{\perp}(f)=0.085 \frac{4 \pi f^{2}}{\rho V_{s}^{3}}|F(f)|^{2}
$$

that is only $7 \%$ less than in unlimited medium.

As a vibrator transmitting force $F$ to the platform fixed on the mine's bottom it is advisable to use reactive mass supported by springs situated between the reactive mass and the platform (fig. $8 \mathrm{a}$ ). This allows to radiate seismic waves in resonance regime. For a slow change of oscillations' resonance frequency one might accordingly change the length or rigidity of springs. The reactive mass value may be changed the same way.

The resonance frequency of reactive mass equals to $\sqrt{K / m} / 2 \pi$, where $K$ is the summarized rigidity of springs, $m$ is the oscillating mass.

The range of the mass alteration may be lessened by simultaneous regulation of the mass and the springs elasticity. Technical solution of problem of resonance frequency change in system will be more easy, if a signal with slow change of frequency is radiated, like a long sweep-signal.

The reactive mass oscillating velocity equals to $\stackrel{\circ}{C}=C \cdot 2 \pi f$ where $C-$ is the amplitude of this mass oscillations, and the force upon the punch is equal to

$$
F=m \cdot 4 \pi^{2} f^{2} \cdot C=m \cdot 2 \pi f \cdot \stackrel{\circ}{C}=K \cdot C \text {. }
$$

At $2 \mathrm{~Hz}$ frequency with $C=0.5 \mathrm{~m}$ we get $\dot{C}=6.3 \mathrm{~m} / \mathrm{s}$ and with $m=50 \cdot 10^{3} \mathrm{~kg}, F=396 \cdot 10^{4} \mathrm{~N}, K=791 \cdot 10^{4} \mathrm{~N} / \mathrm{m}$. Force $F$ remains constant within all the range of frequencies if $K \cdot C$ is constant, too.

The platform displacement

$$
U=F \frac{1+3\left(V_{s} / V_{p}\right)^{3}}{6 \pi a_{0} \rho V_{s}^{2}}
$$

in this example with $\lambda=\mu$, radius of the platform $a_{0}=1 \mathrm{~m}, \rho=2.5 \mathrm{~g} / \mathrm{sm}^{3}$ and $V_{s}=2 \mathrm{~km} / \mathrm{s}$ at $2 \mathrm{~Hz}$ frequency is equal to $U=3.3 \cdot 10^{-5} \mathrm{~m}$, and the radiating power is equal to $J=0.36$ Watt.

At great distances from the considered radiator with no account to the reflections from component semispace border, radial and transverse components of displacement $U_{r}, U_{\theta}$ and $\sigma_{r r}, \sigma_{r \theta}$ of tension components are

$$
\begin{aligned}
& U_{r}=F \frac{\cos (\theta)}{4 \pi \rho V_{s}^{2} r} e^{i k_{\rho} r}, \\
& U_{\theta}=-F \frac{\sin (\theta)}{4 \pi \rho V_{s}^{2} r} e^{i k_{s} r},
\end{aligned}
$$




$$
\begin{aligned}
& \sigma_{r r}=-i F \frac{\cos (\theta)}{4 \pi r} k_{p} e^{i k_{p} r}, \\
& \sigma_{r \theta}=-i F \frac{\sin (\theta)}{4 \pi r} k_{s} e^{i k_{s} r}
\end{aligned}
$$

where $\theta$ - is an angle between the direction of particle oscillations and the direction to the point of observations, $r$ - is the distance from the source, $k_{s}=2 \pi f / V_{s}, k_{p}=2 \pi f / V_{p}$.

\subsubsection{Radiation of "twister"}

Another type of radiator is a "twister" creating the twisting moment of force $M$ vertically oriented and leading to dislocational azimuthal oscillations of mine's periphery . In such case the following power is radiated [lakovich M:A., 1973]

$$
J(f)=\frac{\pi f^{2}}{12 \rho V_{s}^{3}}\left(k_{s} M\right)^{2}
$$

here $M=F \cdot a$ moment of force, $a$ - mine's radius, so that

$$
J(f)=\frac{\pi f^{2} F^{2}}{3 \rho V_{s}^{3}}\left(k_{s} a / 2\right)^{2} .
$$

The "twister's" diagram of directivity is a torus with zero gap and a vertical axis for $U_{r}$ and $\sigma_{r}$ and the rotation surface with vertical axis consisting of two contiguous spheres for $U_{\theta}$ and $\sigma_{r \theta}$. As multiplier is $\left(k_{s} a / 2\right)^{2} \ll 1$ then the inadmissibly large force $F$ is needed to make the "twister" compete with concentrated force despite of the fact that the radiator here may theoretically be of vast dimensions in height.

\subsubsection{Radiation of pulsating sphere}

The radiation power of pulsating sphere is equal to

$$
J_{M}(f)=\frac{\pi f^{2} \rho}{2 V_{p}}|\bar{U}|^{2}
$$

where $\bar{U}$ - its volumetric velocity. The force of medium reaction is an integral of pressure from the side of medium around the radiator surface. In case of pulsating sphere this force is

$$
F=-\left.4 \pi a^{2} \sigma_{r}\right|_{r=a}
$$

where $a$ - the sphere's radius, $\sigma_{m \mid r=a}$ a component of tension upon its surface. 
$\sigma_{r r r=a}=-i \bar{U} \frac{\rho V_{s}^{2}}{2 \pi^{2} f a^{3}}$

There from

$$
F=i \bar{U} \frac{\rho V_{s}^{2}}{\pi f a}
$$

and (3) may be presented as

$$
J_{M}(f)=|F|^{2} \frac{\pi^{3} f^{4} a^{2}}{8 \cdot \rho V_{p} V_{s}^{4}}
$$

Comparing this result with single force radiation described by formula (1) we get

$$
J_{M}(f)=\frac{3}{2+\left(V_{s} / V_{p}\right)^{2}} \frac{4 \pi^{2} f^{2} a^{2}}{V_{p} V_{s}} J(f) .
$$

At about $1 \mathrm{~Hz}$ frequency and $a \cong 3 \mathrm{~m}$ for all real media $J_{M} / J$ is about $10^{-6}$. Thus the radiation to the mine's walls thrust that correspond to that of radiating sphere is not expedient.

\subsubsection{Radiation of centrifugal force circular motion}

The mass circular motion along the mine's surface or the circular tunnel is acting upon the medium with centrifugal force $F=m \cdot 4 \pi^{2} f^{2} \cdot a$, where $a$ is the circular trajectory radius, $m$ - the mass (fig. $8 \mathrm{~b}, \mathrm{c}$ ). From the point of view of oscillation theory the circular motion is the oscillations polarized along the circle which are equivalent to two linearly polarized oscillating processes by two circle diameters perpendicular to each other. Thus the influence of mass circular motion is equal to the effect of two harmonious amplitude forces $F=m \cdot 4 \pi^{2} f^{2} \cdot a$ acting along two mutually perpendicular diameters of circular trajectory with shift of oscillation phases to $\pi / 2$, one about another, that is force $F$ acting with phase shift $\pi / 2$ into two mutually perpendicular directions. Such forces do not influence the radiation of each other, there radiation summarize their power so that such a pair of forces radiate together twice as much as one force.

The summarized acoustic yield of such radiation is equal to

$$
J(f)=\frac{4 \pi^{3} f^{6}}{3 \rho} \cdot m^{2} a^{2}\left(\frac{2}{V_{s}^{3}}+\frac{1}{V_{p}^{3}}\right) .
$$


For example, at $2 \mathrm{~Hz}$ rotation frequency for the mass of about $m=50 \cdot 10^{3} \mathrm{~kg}$ moving circularly along the mine's walls $3.6 \mathrm{~m}$ in diameter $F=7.1 \cdot 10^{4} \mathrm{~N}$ with the velocity of movement $2 \pi f a=22.6 \mathrm{~m} / \mathrm{sec}$.

The corresponding radiation power in longitudinal wave is 0.2 Watt.

The direction characteristics of radiation created by the centrifugal force influence upon the medium is torus with zero gap and vertical axis for $U_{r}, \sigma_{r}$, and for $U_{\theta}$ and $\sigma_{r \theta}$ it is the rotation surface with vertical axis consisting of two contiguous spheres.'

\subsection{Comparison of different types of vibrators}

The comparison of different types of vibrators radiation power showed that two of them are technically acceptable: the type of single force applied to some point of the space, that is to the platform, and the type of rotating force.

The source of the type "pulsating force" is the most close by the type of action to the explosion source, however, its prospect usage is still doubtful. This source version is a couple of forces applied to the mine's or tunnel's walls. In this case the creation of great forces by comparatively simple tool without inertial mass significantly facilitates. Probably just such source is useful for the radiation of high frequency oscillations of $30-40 \mathrm{~Hz}$. As a drawback of such source there should be also mentioned high sensitivity of wave field generated by it in ratio to medium inhomogeneities near it. It happens because the field in a distant zone is a superposition of fields created by each force. These fields are of very similar form but are of opposite phases. That is why the resulting field is a difference of two very alike fields and strongly depends upon each factor distorting the form of each field. This remark is true for explosions either. The increased sensitivity of field towards the medium inhomogeneities near the charge should tell itself at the explosion. This reason points on the expediency of single force and circulating force type vibrators usage.

Vibrator creating turning moment of forces attracts the attention of geophysicists by the fact that such source generates only transversal waves. Whatever alluring the idea is, the "twister" has the same drawback as the source of pulsating sphere type.

Taking in account the necessity of wave generation of substantially larger dimensions than the source, the possibility of such vibrator application should be looked upon as having no prospects.

So, let us look in detail upon the source of the focused type force and the force of circulating type.

\subsubsection{Force applied to the platform}

Vibrators of such type are widely spread in seismic prospecting. They are intended for the solution of problems concerning the study of depths from some 
dozens of meters up to dozens of kilometers. Frequency band is from $6 \mathrm{~Hz}$ up to $200 \mathrm{~Hz}$, force amplitude is from several tons up to 30 ton. These vibrators use hydraulic drive moving inertial mass. Such principle choice is due to several points: the requirements to hard control of initial mass movement, the possibility of quick disarrangement of its oscillations frequency, the convenience of its exploitation in a mobile version. One of the drawbacks of this vibrator is the absence of energy recuperation in it: the energy given to reactive load during the first half of the cycle do not return into the system during its second half. That is why the vibrator consumes enormous yield from the outer source.

The requirements to the UNE's calibration vibrator are quite different. It should radiate seismic energy of relatively low frequency, which demands the creation of large force of pressure upon the platform of hundreds and possibly thousands tons. The demand of inertial mass movement managing becomes less, but the demand of quick frequency disarrangement eliminates, so the use of hydraulic drive becomes obligatory.

As the UNE calibration vibrator should work in stationary regime it will simplify the problem of its design. To achieve greater force there might be used larger mass, perhaps, up to one thousand ton and even larger. Specific exploitation conditions - in the mine, broad bore-hole or gallery give some restrictions to its dimensions and, as it seems, to maximum weight of its part.

The rejection of hydraulic drive allows to solve the question of recuperation of energy. This may be achieved either with the help of two rotating masses the center of gravity of which is displaced in ratio to rotation, or with the help of springs used for resonance concordance, as it shown in part 4.1.

In principle, there may be used even rotating masses and springs. Such technical solution needs special analysis.

Rotating masses are applied in powerful 100 -ton vibrators designed by Siberian geophysicists. At one time, about 15 years ago the question of powerful vibrator type choice was widely discussed. The choice of this very type was done on the base of experimental estimations made by several experts and as it is seen now, was good. The attempt to put this vibrator on pneumatic springs for the resonance regime of work failed due to partly technical causes and do not discredit the plan of future application of this idea.

For the elaboration of vertical vibrator with rotating masses the mine of a round form creates certain difficulties. It is much more easier to design a vibrator producing horizontal force because it suits more naturally such cylindrical room.

Other type of powerful seismic vibrator with hydraulic drive was worked out in Gomel Design Bureau "Seismotechnic", Belorussia. In essence it employs the same type of hydraulic drive that the seismic prospecting vibrators but produces greater force, up to 100 - ton. This vibrator uses very heavy inertial mass about 150 - ton, which is fixed upon elastic supports. In Gomel engineers' opinion, the powerful vibrators employing hydraulic drive are most prospective for further development.

Unfortunately, the experiments on this vibrator application for the Earth's deep entrails sounding were stopped at the beginning because of the "perestroyka", 
the disintegration of the Soviet Union and disestablishment of Belorussia into a separate state.

Let us look upon the seismic radiation power formula

$$
\begin{aligned}
& J(f)=\frac{\pi f^{2}}{6 \rho V_{p}^{3}}|F(f)|^{2}+\frac{\pi f^{2}}{3 \rho V_{s}^{3}}|F(f)|^{2}, \\
& F(f)=m \cdot 4 \pi^{2} f^{2} C .
\end{aligned}
$$

The first item characterized the radiation yield into longitudinal wave $L p$, the second one - into transversal wave $L s$. These yields ratio with Lame constants equality is $J_{p} / J_{s}=V_{s}^{3} / 2 V_{p}^{3}=1 / 10.4 \equiv 0.1$.

Thus only $9 \%$ of total seismic yield radiates into longitudinal wave. If we assume $V_{p} / V_{s}=\sqrt{3}, V_{p}=3500 \mathrm{~m} / \mathrm{sec}, \rho=2500 \mathrm{~kg} / \mathrm{m}^{3}$, that will correspond to consolidated sediments.

Lets make two extreme estimations:

one for force which is necessary to produce for the development of such yield that could radiate longitudinal seismic wave energy equal to UNE's energy of $1 \mathrm{kt}$ $5 \cdot 10^{9}$ Joule, during 24 hours; and the other is the amount of energy that could be radiated by a vibrator into longitudinal wave if it develops 1000 ton force (maximum from modern point of view) and if it radiates seismic wave into medium for two weeks in all(which seems to be top duration).

The table 2 shows the results of estimations.

Table $2 E=5 \cdot 10^{9}$ Joule

\begin{tabular}{|l|c|c|c|c|c|c|c|c|c|}
\hline \multicolumn{10}{|c|}{$E=5 \cdot 10^{9}$ Joule } \\
\hline$f, \mathrm{~Hz}$ & 1 & 1.5 & 2 & 3 & 5 & 7 & 10 & 15 & 30 \\
\hline$F$, ton & 352 & 234 & 176 & 118 & 70 & 50 & 35 & 23 & 12 \\
\hline$F=1000$ ton \\
\hline$f, \mathrm{~Hz}$ & 1 & 1.5 & 2 & 3 & 5 & 7 & 10 & 15 & 30 \\
\hline$J$, Watt & 0.47 & 1.1 & 1.9 & 4.2 & 12 & 23 & 47 & 106 & 422 \\
\hline$E, 10^{6}$ Joule & 0.56 & 1.3 & 2.3 & 5.0 & 14 & 28 & 56 & 121 & 504 \\
\hline
\end{tabular}


As it is seen from the table the radiation with the help of vibrator of low frequency seismic energy corresponding to $1 \mathrm{kt}$ energy of underground nuclear. explosion is technically impossible task. To fulfill such task it is necessary either to radiate for a too long time, or to make a more powerful vibrator. The fulfillment of the last requirement is blocked by the dimensions of the mine, tunnel. One may choose grouping and set not one but several sources. In any case such model might turn out to be technically expedient.

Our estimations of UNE's seismic energy radiated in frequency band of $1-2.5 \mathrm{~Hz}$ may be wrong, possible increased. To make a more exact estimations it is necessary to held special investigations.

Let's mentions that the formula for $J p$ evaluation is true for any orientation of force in space. It is possible to create a three-component vibrator and by way of proper signals processing to synthesize seismograms corresponding to every direction of force.

\subsubsection{Vertical force applied to the surface}

There is no limitation in dimensions for a surface vibrator. That makes possible the creation of more powerful sources by grouping of a greater number of vibromodi. It is more easy technically to use surface vibrators producing vertical force whereas for horizontal vibrators special devices are needed to fix the platforms. The problem only seems to be easy, actually its technical solution is bound with creation of special platforms, anchors and a vast amount of knowhow.

It is expedient to make up a low frequency surface vibrator from vibromoduli of $100-200$ ton.

That will need the usage of large masses to fix the platform upon the surface. The Siberian geophysicists have worked out a low frequency vibrator utilizing water swinging upon the air spring as an inertial mass.

The vibrator scheme is shown on fig. 8 . The vibrator is a vertically set tank filled with water. In the lower part of the tank there are air reservoirs with rhythmically increasing and decreasing air pressure.

The surface vibrator's total radiation yield is 0.93 of the yield of vibrator fixed in a deep mine. For all that, unlike the last one the surface vibrator radiates not only longitudinal and transversal, but a Raleigh wave as well, and it is only $7 \%$ of total yield that falls on longitudinal wave. Force $F$ acting upon the surface produces longitudinal wave the yield of which constitutes $72 \%$ from the $P$-wave yield produced by similar force in the mine. This is close enough value, so it is possible to use the table in estimation of surface vibrator possibilities.

The table like the previous one shows force that should produce the vibrator set on the surface to radjate within 24 hours the longitudinal wave energy of $5 \cdot 10^{9}$ Joule and the yield of longitudinal wave which it will radiate within two weeks at pick force 1000 ton. 
Table 3

\begin{tabular}{|c|c|c|c|c|c|c|c|c|c|}
\hline \multicolumn{1}{|c|}{$E=5 \cdot 10^{9}$ Joule } \\
\hline$f, \mathrm{~Hz}$ & 1 & 1.5 & 2 & 3 & 5 & 7 & 10 & 15 & 30 \\
\hline$F, 10^{3}$ ton & 417 & 270 & 202 & 130 & 78 & 57 & 40 & 27 & 15 \\
\hline$F=1000$ ton & 1 & 1.5 & 2 & 3 & 5 & 7 & 10 & 15 & 30 \\
\hline$f, \mathrm{~Hz}$ & 0.42 & 0.97 & 1.7 & 3.7 & 10 & 21 & 42 & 91 & 378 \\
\hline
\end{tabular}

The parameters in this table are rather close to those in the previous one. However, if for mine vibrator we shall take the maximum force to be equal to 100 ton, than for surface vibrator it is possible to accept a more optimistic estimation of 500 ton. That rather changes the course of events.

Thus, to calibrate the UNE's yield at frequencies lower than $10-15 \mathrm{~Hz}$ it is expedient to apply surface vibrator, and for calibration at higher frequencies - a mine vibrator.

The application of surface vibrator for UNE's yield calibration is bound with at least two methodological problems: inhomogeneity under vibrator and dislocation of vibrator in ratio to the hypocenter of explosion.

Ground inhomogeneity usually tells in a form of stratified structure. If elastic characteristics of layers are known, the problem on the whole may be solved in flat-stratified ideal elastic medium approach. Stratified structure creates mechanical resonance that may considerably hamper the calibration problem. Radiated field of longitudinal waves may be controlled in a bore-hole under. vibrator and as a sounding signal is radiated by source it seems expedient to use the signal registered by seismograph installed in a bore-hole.

The influence of vibrators dislocation towards the hypocenter of explosion may be different and depends on inhomogeneity of the medium. If the medium is homogeneous such influence is relatively small but it would be much more prominent if the medium turns out to be inhomogeneous, particularly laterallyinhomogeneous. In such case there would remain an unregistered error that could be estimated and accounted for by theoretical calculations if the structure of the Earth crust uppermost part is known.

On the basis of common considerations it is clear that this error's value directly depends on frequency and it may prove to be insignificant for low frequency. 


\subsubsection{Cenrifugal force. Vibrator in the shaft}

Centrifugal force is moving along the circle. A vibrator based on this principle an inertial mass moving along the wall of circular cylinder - a gallery, a mine. Interest to such type of vibrator is due to the fact that its construction may be well fitted to a steep mine in which nuclear device is lowered. An inertial mass itself may be fixed on "electric automobile" on "electric train" that will move along vertical side of the mine as it is shown of fig.9.

It is evident that actually the construction might be quite different with no semblance to automobile or train at all. As the advantages of such source may be treated its simplicity and the ability to provide good contact with mine's wall, soil, because the centrifugal force should tightly press the vibrator's surface to the mine's wall.

Seismic power radiated by such vibrator is equal to

$$
J(f)=\frac{4 \pi^{3} f^{6}}{3 p V_{p}^{3}} m^{2} a^{2}+\frac{8 \pi^{3} f^{6}}{3 p V_{s}^{3}} m^{2} a^{2} .
$$

The first item is the power radiated into longitudinal wave, the second item - that into transversal wave. Suppose, like it has been before, that Lame's constants are equal and $V_{p}=V_{s} \sqrt{3}$.

Radiation power ratio into longitudinal and transversal wave is

$$
J_{p} / J_{s}=V_{s}^{3} / 2 \cdot V_{p}^{3}=0.10 \text {, }
$$

the same as to a vibrator acting as force applied to an inner point of the medium. Let us suppose as it was in the previous case, that $V_{p}=3500 \mathrm{~m} / \mathrm{s}$, $\rho=2500 \mathrm{~kg} / \mathrm{m}^{3}$. The estimation of force that should be developed by centrifugal vibrator to radiate within 24 hours the longitudinal wave energy of $5 \cdot 10^{9}$ Joule and the estimation of vibrator's energy which it will radiate into longitudinal wave within the two weeks work with pick force equal to 1000 ton, are given in the table. 
Table 4

\begin{tabular}{|l|c|c|c|c|c|c|c|c|c|}
\hline \multicolumn{1}{|c|}{$E=5 \cdot 10^{9}$ Joule, } \\
\hline$f, \mathrm{~Hz}$ & 1 & 1.5 & 2 & 3 & 5 & 7 & 10 & 15 & 30 \\
\hline$F, 10^{3} \mathrm{kt}$ & 303 & 165 & 125 & 80 & 50 & 35 & 25 & 16 & 9 \\
\hline \multicolumn{10}{|c|}{16} \\
\hline$f, \mathrm{~Hz}$ & 1000 ton, & 1.5 & 2 & 3 & 5 & 7 & 10 & 15 & 30 \\
\hline$E, 10^{6}$ Joule & 1.1 & 2.6 & 4.6 & 10 & 28 & 56 & 112 & 242 & 1008 \\
\hline
\end{tabular}

As we can see, with the same force amplitude as a vibrator creating harmonic force applied to the medium's inner point, the centrifugal vibrator possesses greater yield and radiates much more energy into longitudinal wave within the same time.

This fact is bound up with centrifugal vibrator producing continuous maximum force while the vibrator of focused force type produces variable force and its quadratic mean is 1.4 times less than maximum.

It seems to be expedient to use the centrifugal type vibrator in a mine and not on the surface. Setting on the surface seems unsuitable because the characteristic of $\mathrm{P}$-wave direction in departure angle $i$ function has minima at $i=90^{\circ}, \quad i=0^{\circ}$ and a complicated form depending on structure of uppermost part of sediments. Another difficulty is due to the soil being relatively easy destroyed by share stresses and more stable towards normals stresses.

When exploiting the horizontal centrifugal vibrator within the medium the diagram of directivity does not depend on radiation amplitude and depends on departure angle as $\sin i$.

$P$-wave registered at $200-1000 \mathrm{~km}$ distance from the source has departure angle $i=20-25, \sin i=0.3-0.4$.

It means that radiation by horizontal force is less advantageous than by vertical force, because in case of vertical force the diagram of directivity depends on departure angle as $\cos i$, which is $0.94-0.91$ with $i=20^{\circ}-25^{\circ}$. In the end, the choice of the type of vibrator should be dependent not only on longitudinal wave radiation effectivity but on technical advantages as well. 


\subsubsection{Seismic power of vibrator for UNE calibration}

Estimations adduced in the previous point show that the creation of a vibrator that would be able to radiate within the real time(of 2 weeks) seismic energy equivalent to that radiated by UNE of $1 \mathrm{kt}$ yield, is impossible, or bound with enormous technical difficulties.

In this connection there at least two questions arise:

- whether estimations obtained by us contradict the results of $100-$ ton vibrators seismic yield measurements;

- at what seismic yield should be orientated the creation of seismic vibrators.

As it was noted before, the 100 ton seismic vibrators radiate the power of about 100 Watt into longitudinal wave at $5-10 \mathrm{~Hz}$ frequency. It is essentially 12 times more than the power which, in our estimation, will produce a 1000 ton vibrator at $5 \mathrm{~Hz}$ frequency, and correspondingly 1200 time more a 100-ton vibrator will produce. The cause of it lies, first of all, in the difference of medium's parameters. Sedimentary layer on which both vibrators are set, the Novosibirsk and the Baikal one, is characterized by velocity value $V_{p}=700 \mathrm{~m} / \mathrm{sec}$, density $\rho=1500 \mathrm{~kg} / \mathrm{m}^{3}$.

The due regard of these differences lessens the discrepancy of estimations for about 220 times. There remains the discrepancy of about 5.5 times, which is possibly connected with inaccuracy in estimation of vibrator seismic radiation power and with inhomogeneity of the uppermost part of sediments.

It is difficult to decide now which of the causes have greater importance, because the question has not been specially studied. However, there are some reasons to suppose that vibrator's seismic power is determined not enough exactly.

Total seismic power is determined by formula

$$
J=\stackrel{\circ}{U} \cdot F \cdot \cos \phi
$$

where - $\stackrel{\circ}{U}$ the velocity of ground displacement under the platform of vibrator. $F$ force amplitude, $\phi$ - phase shear between $\stackrel{\circ}{U}$ and $F$. With vibrator's work on the sedimentary soil surface in the zone of large deformations near the vibrator (up to $30-50 \mathrm{~m}$ ) there is observed typical nonlinear distortion of oscillations. It is bound with nonlinearity of stress - strain diagram expressed in difference of elastic moduli for compression and tension deformations. Actually, moduli of volumetric deformation are some tens percent more for compression than for tension. As the consequence of such difference the particle displacement upwards is more than displacement downwards [Nikolaev A.V.,1967]. Thus there forms constant deformation of tension, especially strong under vibrator's platform [Vasiljev Yu.I. et al., 1986]. 
So, the value under the vibrator's platform is increased by nonlinear conduct of soil and do not correspond to particle velocity value produced by force $F$.

To eliminate such error it is necessary to determine particle velocity in a shallow bore-hole of $30-50 \mathrm{~m}$ under the vibrator.

Sediments at the depth of several hundred meters are much more consolidated than near the surface which tells in relatively large values of seismic waves velocities. At the same time vibrator's seismic power greatly varies according to seismic wave velocity in the medium near vibrator: double increase of velocity corresponds to 8 times power decrease!

It also points to the expediency of surface vibrators usage, first of all, the low frequency ones.

The question of optimal amount of energy that should be radiated by vibrator for UNE calibration is in the end reduced to signal to noise ratio for $P$ wave on a vibro-pulse seismogram. The main hindrance is microseism. Microseisms level varies both in space and time. Temporal variations of microseisms level is bound with time of the the day, season, weather and are often more than $10 \mathrm{~dB}$. Spatial variation between most quite registration points and those with medium intensity of microseismic background are about one order to a low frequency zone of $1-5 \mathrm{~Hz}: 3 \cdot 10^{-9}-3 \cdot 10^{-8} \mathrm{~m} / \mathrm{sec}$.

In further calculation we shall orientate ourselves to $10^{-8} \mathrm{~m} / \mathrm{sec}$ level corresponding to good registration conditions, and to calibrated signal to noise ratio 10 . With such conditions calibrated signal's energy might significantly reduce.

The following table 5 shows how many times might be decreased the energy of calibrated signal corresponding to $1 \mathrm{kt}$ yield explosion to achieve the signal to noise ratio 10 at different epicentral distances, as well as the amount of energy determined on base of the evaluation $E=5 \cdot 10^{9}$ Joule, corresponding to yield of $1 \mathrm{kt}$.

Table 5

\begin{tabular}{|l|c|c|c|c|c|c|c|c|}
\hline Distance, $\mathrm{km}$ & 300 & 400 & 500 & 600 & 700 & 800 & 900 & 1000 \\
\hline $\begin{array}{l}\text { Energy } \\
\text { reduction }\end{array}$ & $1.6 \cdot 10^{-5}$ & $5.6 \cdot 10^{-5}$ & $2.1 \cdot 10^{-4}$ & $9.2 \cdot 10^{-4}$ & $2.8 \cdot 10^{-3}$ & $1.0 \cdot 10^{-2}$ & $4.3 \cdot 10^{-2}$ & $1.0 \cdot 10^{-1}$ \\
\hline $\begin{array}{l}\text { Energy, } \\
10^{6} \text { Joule }\end{array}$ & 0.065 & 0.23 & 0.90 & 3.8 & 11.5 & 41 & 170 & 412 \\
\hline
\end{tabular}

Comparison of data from this table with tables 3 and 4 shows that low frequency vibrator developing 1000 ton vertical force in a mine or on the 
surface, within 2 weeks could radiate into seismic longitudinal wave enough power to calibrate UNE with following registration characters. For $1 \mathrm{~Hz}$ frequency at distance up to $300 \mathrm{~km}$, for $2 \mathrm{~Hz}$ frequency at distance up to $600 \mathrm{~km}$, for $7 \mathrm{~Hz}$ frequency at distance up to $800 \mathrm{~km}$ from the source. Signal to noise ratio then would be average, not less than 10 if microseisms level is not higher than $10^{-8} \mathrm{~mm} / \mathrm{sec}$.

The adduced calculation gives approximate estimation of UNE's seismic calibration possibility by vibrator when registration is held by singular vertical seismometer. It is evident that this method possiblities can be improved by special modes of noise suppression: usage of three component registration and polarizational filtering; frequency filtering, usage of microarray, consisting of $10-20$ seismometers, aperture up to $1-2 \mathrm{~km}$.

The effectivity of these actual signals registration methods depends on field of microseisms structure and may in average give 2-3 times improvement of signal to noise ratio. This corresponds to possibility of decreasing seismic vibrator radiation energy into $4-9$ times.

Thus, the low frequency vibrator producing $1000 \mathrm{t}$ force might solve the problem of UNE calibration in a frequency band of more than $1 \mathrm{~Hz}$ with observations at up to $1000 \mathrm{~km}$ distances even with rather high seismic impendance of rocks defined by value of $V_{p}=3500 \mathrm{~m} / \mathrm{sec}$ and $\rho=2600 \mathrm{~kg} / \mathrm{cm}^{3}$. Another medium parameters will give another energy estimations and, correspondingly, another main parameters of vibrator - pick force and the duration of sweep - signal.

A useful way of vibrator design is modulus principle: the usage of several vibromoduli close situated and working synchronously. Proceeding from the obtained experience it is possible to advise moduli developing force up to 50-100 ton. Grouping of moduli may be done in a mine as well as on the surface. Another important characteristic of vibrators - the frequency of velocity changes - may be low enough as it is planned to use long sweep-signals. This makes it possible to use rotating masses as a force device and resonance matching of vibrator and medium by means of springs with variable rigidity.

To get low frequency (up to $10 \mathrm{~Hz}$ ) and high frequency (more than $10 \mathrm{~Hz}$ ) waves it is advisable to make separate vibrators. The question needs special investigations. In particular, it is necessary to evaluate seismic energy required to calibrate UNE at 10 to $40 \mathrm{~Hz}$ frequencies.

\subsection{Powerful seismic vibrators: State of elaboration of the problem}

For a number of year, from 70-s up to 90-s several research groups elaborated powerful seismic vibrators for investigation of the Earth interiors and for seismic monitoring. Those groups applied different principles of force device creation on the basis of inertial mass movement. The result of these works is 
elaboration and testing of several types powerful vibrators and their low-powered models. Another important result of the works is the formation of small groups of engineers and geophysicists experienced in elaboration and management of powerful seismic vibrators. Each group is headed by chief designer and works out types of sources. The main groups have formed up till 1980, nowadays their work is on the whole stopped though the groups still remain.

Here are the main groups, directions of their researches and state of investigation of the problem.

1. Institute of Geophysics of Joint Institute of Geology, Mineralogy and Geophysics of Siberian Branch of RAS. Investigation leaders are Prof. I.S.Chichinin and Prof. V.I. Jushin. There have been elaborated a number of "debalanced" vibrator models based on inertial masses rotation principle, the gravity center of which is displaced towards the rotation axis. These vibrators produce maximum centrifugal force of 5 ton, 40 ton, 100 ton. Low-powered debalanced vibrators have frequency range up to $80 \mathrm{~Hz}$. They are designed for seismic prospecting in Siberian conditions (work in taiga almost inaccessible regions, cold winter), they are the basis of seismoprospecting complex "Vibrolocator". The Siberian Experimental Design Bureau has produced about 10 copies. Low-powered vibrators were used for model investigations, particularly, for the study of "vibrator - soil" system resonance characteristics, for the study of harmonic oscillations field structure created at vertical and horizontal action, at various distances from the source, the study of conditions of placing the vibrator (sediments, upper part of the soil freezing) upon stability of radiated signal. 40 ton stationary debalanced model of a vibrator [Chichinin I.S.] was designed and tested before a more powerful, 100 ton vibrator was made, systems of stabilization and control of mass rotation were studied on this model.

The first 100 ton vibrator was made in 1978, its drawbacks were not enough stable control upon mass rotation and direct dependance of force square frequency. Both drawbacks were eliminated later. Regulation of force according to rotation frequency is performed by change of distance between mass gravity center and rotation axis.

Two new 100 ton vibrators were set on Bystrovka test site near Novosibirsk and about settlement Babushkin near Lake Baikal (fig.9). But for the mentioned results of registration at $300 \mathrm{~km}$ distance from the source, the experience of work on Novosibirsk vibrator has shown that at $500 \mathrm{~km}$ the $P$-wave is singled out enough distinctly on a vibro-pulse seismogram. With a vibrator working in harmonic regime (1 hour of continuous work), the harmonic signal is distinctly detected on noise background at $1100 \mathrm{~km}$ distance from the source.

The experience of work on the Baikal vibrator has shown that at $125 \mathrm{~km}$ distance from the vibrator the precision of determination of $P$-wave maximum phase arrival time towards the source is not less than $0.001 \mathrm{sec}$. Such high precision is achieved due to high stability of vibrator's work and to high signal to noise ratio. 
Debalanced vibrators have relatively simple construction, are put in motion by standard electric engines and have good resource of continuous work. They are usually exploited in conditions of separate seances with 40-60 min duration during which a sweep-signal or harmonic signal is radiated. There is a possibility of producing both simple sweep-signals with linear change of frequency in time and complicated sweep-signals with non-linear change of frequency as well.

Nowadays both vibrators are preserved with sharp decrease of financing.

The Laboratory of the Earth vibrosounding of the Institute of Geophysics headed by prof. V.I.Jushin has in its staff engineers and scientists of high professional skill, it has vast experience in creation and investigation of vibrational sources, in creation of special equipment for data processing.

This group may play first fiddle in creation of powerful vibrator for UNE calibration.

As the shortcomings of the leaders may be treated their negative attitude towards the possibility of creation of powerful vibrators based on other technical principles.

2. Computing Center of the Siberian Branch of RAS. Investigation leaders: the director of the Center academician A.S.Alexejev and head of Laboratory V.V.Kovalevsky. During some years (1978-1993) the Computing Center closely cooperated with the Institute of Geophysics and held investigations in the field of elaboration of algorithms and programs of vibrational signals processing, creation special equipment for data registration and processing. Since 1986 the Computing Center has started independent elaboration of a vibrator which uses oscillating movements of water upon air spring.

The idea was proposed by V.V.Kovalevsky who first realized it in a small model ( 3 liters of water), then in a 60 ton and 100 ton vertical vibrators, 250 ton horizontal vibrator., shown on the fig. 10.

As it was stated above, vertical vibrator is a vertically set tank of a railway carriage into which $120 \mathrm{~m}^{3}$ of water is poured. In the lower part of the tank there are air cells. The air is compressed when the water moves down and expands when it moves upwards. Air cell volume may change, accordingly changes air spring rigidity and system's own frequency. Horizontal vibrator works on the same principle. Horizontal tube is filled with water, the air is in its two ends turned up as is seen on fig.10. Horizontal vibrator consists of four separate elements working synchronous.

The expedience of this direction development is not yet quite evident. In the authors' opinion such vibrator should be used only in low frequency regime, up to $10 \mathrm{~Hz}$.

The vibrator is set on a test site near Novosibirsk. Its testing has shown that having equal pick force with a debalanced vibrator it radiates approximately the same power. The authors of this work think that the vibrators advantages are the simplicity of its manufacturing and the simplicity of mounting - dismounting and transportation, because the water which is the working mass might be poured in on the very place of work and be poured out before the vibrator's transportations. 
Another advantage is the relative simplicity of operating, the resonance regime of work, relatively low energy consumption.

Mechanism of operation is worked out and made in the Design Bureau of the Computing Center. The organization has normal possibilities of mechanical works, so the very fact of successful elaboration of operating mechanism testifies to its simplicity and to safety of its structure.

Being unusual both in principle of functioning and in form this source has not yet won enough sympathies between investigators - geophysicists and engineers. A small group of engineers - elaborators has in view the creation of even more powerful sources functioning on the same principle. A model version of such vibrator may be a much more powerful vibrator in which a ballistic missiles mine is used as a tank for water. Now, the reduction of missile armaments and destruction of missile mines takes place in Russia. The work on design and testing of such vibrators, including unique in dimensions and power, might be performed by the staff of the Laboratory of vibrational seismic investigations of the Computing Center. To perform this task the staff has the necessary experience, both scientific and technical, possibility of designing such mechanism and electronics in a Design Bureau.

Now, the works on testing the available vibrators are stopped.

3. Gomel Special Design Bureau SDB. "Seismotechnique" Gomel, Belorussian Republic. The SDB "Seismotechnique" was founded at the beginning of 70 for elaboration of non-explosive sources for seismic prospecting, seismodins and vibrators. Up till 1988 the investigation leader was prof. Shaginjan, after 1988 - A.G.Asan-Dzalalov. The main direction is elaboration of hydraulic vibrators for seismic prospecting similar to those produced by firm "Mertz", "Prakla-Seismos", company "CONOCO" and others. The same principle was used in production of increased power vibrators: a 30 ton mobile and a 100 ton stationary.

A 30 ton mobile vibrator shown on fig. 11 was made in 1988 to order of the Institute of Physics of the Earth AS for scientific research aims. It is used for study of nonlinear phenomena of seismic waves spreading and or study of vibroaction influence upon productivity of oil deposit and on effect of induiced acoustic emission.

Stationary 100 ton seismic vibrator shown on fig.12 designed for seismic monitoring of 'the Earth crust is an inertial mass about 150 ton supported by bearing. Each of bearings has small platform stated upon gravel bolster which serves as a spring. Hydrochamber is stiffly bound with inertial mass, and piston is bound with the Earth through the platform. Frequency band is $2-50 \mathrm{~Hz}$. The conditions of vibrator's setting turned out to be unsuccessful because of semisandy soil liquification under strong vibrations. To fight these phenomena various measures had to be constantly taken, in particular, gravel was added, exploitation was held not at full strengh, metallic piles were used to strengthen the soil. The obtained experience is useful for the right choice of vibrators setting conditions. 
SDB "Seismotechnique" has a test site equipped with seismic vibration measurement technique. In particular, there is a $900 \mathrm{~m}$ bore-hole in which seismic receivers for determination of pulse and vibrational radiation yield are set every $100 \mathrm{~m}$.

Technique elaborated in SDB is produced by "Seismotechnique" factory which have enough possibilities to make hundreds of seismic sources in a year. SDB has very qualified designing staff orientated at elaboration of solely hydraulic and pneumatic sources, vibrators and seismodins. These sources quality is up to international standards.

The engineering personel that created powerful source has high professional skill and creative potential that might be further used at elaboration of powerful seismic vibrators of hydraulic type. In principle, the SDB and the factory have possibilities of creation of quite different vibrators types. However, between the leading designers there dominates an opinion that hydraulic vibrators have such prominent advantages in comparison with vibrators of other types that there is no need to elaborate any other ideas.

A 100 ton vibrator is for a number of years in a non-working state, though it may be quickly reanimated if necessary. Despite of slump the EDB's is in a good enough form to carry on active work upon the creation of powerful hydraulic vibrators.

4. The Joint Institute of Physics of the Earth of RAS. The works are concentrated in the laboratory of experimantal geophysics, the head of the laboratory Prof. A.V.Nikolaev. For the space of many years, since 1975, the Institute performed coordinative functions upon the works and was the executor of geophysical researches upon the problem "Vibrational sounding of the Earth", it played the part of a customer of powerful vibrosources on the initial stage of investigations. The laboratory personel had started a number of pioneer works which were later developed in other organizations. In particular, there were elaborated methods of seismic tomography using harmonic regime of work of seismic source (this work was also carried out quite independantly by Computing Centre of Siberian Branch of RAS); were found out the interactions of intensive seismic wave field with other geophysical fields - magnetic, electric, seismoacoustic; was found seismoradiational effect and the origination of constant expansion deformation near vibrator; was found out effects of weak seismic vibrations influence upon oil productivity; were studied nonlinear seismic effects - interaction of seismic waves.

Among nonlinear effects there should be singled out one which is of great importance for the creation of powerful low frequency sources. This effect is the detection of wave transmitting through medium with strong non-linearity. In particular, when amplitude - modulated high frequency signal is transmitting there is observed the origination of low frequency seismic signal which frequency is equal to modulation frequency. Sources using such nonlinear transformation of signal are applied in sea acoustics and are called parametrical.

Parametrical radiation at low frequencies has significant advantages over straight radiation, because in this case there may be used high frequency 
sources having greater radiation power even at comparatively small forces. The drawback of this source is that the direction of radiation on one hand is pronounced, and on the other hand it is difficult to control and manage because of inhomogeneity of non-linear features distribution in the upper part of medium.

All the same, the obtained results allow to consider this trend to be perspective. Nowadays, the investigation of parametric radiation is carried out by the Joint Institute of Physics of the Earth together with Scientific - Research Institute of Radiophysical Measurements in Nizniy Novgorod.

The Joint Institute of Physics of the Earth has specialists of high professional skill, having the experience of various investigations connected with vibrational radiation of seismic waves and their propagation though the real medium. This experience may be widely used in scientific researches connected with UNE's calibration problem with the help of seismic vibrators. At present, the staff has the necessary minimum of tools to start investigations: a 30 ton mobile seismic vibrator, sensors for seismic and acoustic measurements, tiltmeters and deformometers for registration on the surface and in a borehole; necessary means for information recording and processing. Leading Russian scientists and engineers in the field of acoustic radiation, elastic waves spreading in non-linear media, problems of physics of explosion and calibration of its yield may be enlisted to this work.

Thus, Russian Academy of sciences has strong enough creative and technical potentialities and experience to immediately start and continue investigations upon elaboration of UNE's calibration method with the help of powerful seismic vibrators.

\subsection{Conclusion}

Our estimations of seismic vibrators radiation power are made for some mean conditions. Similar calculation could be easily done for concrete simple cases (homogeneous ideal elastic medium), more complicated radiation conditions require application of special methods of evaluation.

All the same, the simplified estimation gives clear and general physical idea about the problem of seismic waves radiation by vibrator.

1. Rocks possess high impedance, so to radiate even small power of tens Watts into low frequency seismic oscillations enormous force is needed. With fixed force radiation power greatly changes depending on seismic waves velocity in rocks.

2. At several hundred meters depth rocks are considerably more consolidated than near the surface, so seismic wave radiation from the mine call for a much more greater force than radiation from the surface of the Earth. In a typical case the seismic wave velocity at $500 \mathrm{~m}$ is about two times more than in the upper $50-\mathrm{m}$ layer, density is 1.5 times more. So, to produce identical seismic radiation power the mine vibrator should produce at least 10 times greater force that the surfacial vibrator. This could become a dicisive factor when solving the problem whether to set vibrator in a mine, which is more correct from the point of view of 
method, though more complicated, or to set it on the surface, which is technically more simple though is bound with less accurate determination of medium frequency characteristic and additional error in UNE's yield determination.

3. It seems to be technically unreal or too expensive to create mine vibrator which should radiate 10 Watt yield seismic longitudinal wave at $1-2 \mathrm{~Hz}$ frequency. So it is expedient to perform the calibration of UNE by low frequency part of longitudinal wave spectrum of $5-10 \mathrm{~Hz}$ by furface vibrator. This seems even more reasonable considering that focus depth measured by seismic waves length is comparatively small for long-period waves. The calibration of UNE by high frequency part of spectrum, that is more than $10 \mathrm{~Hz}$ seems reasonable to be done with the help of mine vibrator. However, there are no yet reliable estimations of energy necessary for high-frequency signal calibration, so the question remains unsolved.

4. The analysis of radiation effectivity of sources based upon different principles has shown that the creation of sources without inertial mass producing a pair of forces acting upon opposite walls of the mine, gallery, is technically unreasonable, as it requires the application of too large forces. So, there remains one way out - the creation of vibrators with vertical mass of the type of force applied upon the platform. The demand of large forces creation involves application of large inertial masses, large amplitudes of their movement, large energy consumption. It also set technical limits upon design of mine vibrator, but is not the main difficulty in design of superfitial vibrator.

The obtained experience of elaboration and exploitation of powerful vibrators shows that the reasonable way is the design of standard vibromoduli producing $50-100$ ton force. In each concrete case various amount of moduli might be used. In principle it is desirable to use both vertical and horizontal vibrators. However, the setting of horizontal vibrators upon the surface is bound with solution of a complicated problem of their contact with the soil. Another problem is their "bad" diagram of directivity. Both these facts give grounds for doubts in expediency of horizontal surfacial vibrator usage. Design of horizontal shaft vibrator may turn out to be a more easy problem.

5 . It is reasonable to apply resonance principle of inertial mass oscillations. With change of frequency spring's stiffness and/or inertial mass should change. This way has not yet found proper application though its advantages are evident.

6. Hydraulic vibrator are less advantageous than centrifugal ones from the point of view of energy consumption. At the same time they are more compact, might use considerably greater inertial masses, be better managed and could produce complicated pulse signals. It is important that the technique of their design is well elaborated and mastered. That gives them considerable, and possibly desicive advantage. The problem of choice of vibrator's type needs special study and comperative testing of competing models.

7. The existing experience of development and exploiting of powerful seismic vibrators allows to treat optimistic the problem to be solved while designing UNE calibration vibrators. The most easy and reasonable starting point of investigations is the study of seismic waves radiation process with the help of 
existing powerful seismic vibrators - Gomel's hydraulic and Novosibirsk debalanced ones.

The investigations may be organized on Novosibirsk and Gomel vibro test site. 


\section{Direct calibration of UNE by seismic vibrators: technique and problems}

The main question of UNE direct calibration is connected with different nature of seismic sources which radiate significantly different signals. The seismogram corresponding to the long-lasting sweep generated by vibrator must be processed using special technique which transforms it into the common form. This technique is widely used in seismic prospecting. Here we describe the main idea of application the "Vibroseis" method and discuss the problem of determination of the frequency characteristic of the media.

\section{1. "Vibroseis" method}

"Vibroseis" method is widely applied in seismic prospecting owing to some key advantages over traditional methods using explosions. It is ecologically clean, it makes possible seismic prospecting in populated areas, it does not yield to seismic prospecting in the quality of results.

In the bases of this method there is an idea of "compression", that is the transformation of long seismic vibrosignal into a short vibro-pulse one (fig.13). By means of special mathematical treatment the registered vibrogram converts into vibro-pulse seismogram whence each wave is "contracted" in time with its amplitude increasing. However, the pulse signal from UNE and the vibroimpulse one from the vibrator have certain differences even if the wave field forming is due to linear approach. Let us imagine that a seismic vibrator radiates a long signal of variable frequency - a sweep signal, with displacement in volume wave $1 \mathrm{~Hz} P_{v o}(t)$, its spectrum $S_{v o}(f)$ (here and further on we shall mark by " 0 " and " $C$ " symbols fitting to source or to a point of observations, by " $V$ ", " $V P$ " - the vibrational and vibro-pulse signals accordingly.

Considering that seismic signal passing through the medium is not subjected to frequency distortions and there is no velocities' dispersion, then each individual volume seismic wave in the registration point $C$ should have the form of a sweep-signal, its amplitude $A_{t}$ and arrival time $t_{t}$ are determined by conditions of spreading:

$$
U_{v c}^{\prime}(t)=A_{i} U_{v o}\left(t-t_{i}\right) \text {. }
$$

Actually, an individual signal has undergone the distortion of form on account of energy dissipation dependence on frequency. This question may be specially studied but the consideration of frequency distortions will not change the estimations that would be obtained due to our simplified suppositions.

As the sweep-signal has greater duration $T$, the vibrogram $U_{v c}(t)$ registered in $C$ point is a completely nonresolved picture of separate waves interference:

$$
U_{v C}(t)=\sum_{i} A_{i} \cdot U_{v o}\left(t-t_{i}\right)
$$


Seismic energy. of each signal lies in a prolonged time interval $T$, that is why each signal and vibrational seismogram amplitude is considerably less than the amplitude of pulse signal with equal energy. Vibrational seismogram $U_{V C}(t)$ may be transformed into vibro-pulse seismogram by its correlation with the sweepsignal reversed in time $U_{v o}(-\tau)$ :

$$
U_{v P C}\left(t-t_{t}\right)=\int_{-\infty}^{\infty} U_{v C}(\tau) \cdot U_{v o}(t-\tau) \cdot d \tau
$$

As a result of this transformation, each individual wave having the form of $A_{i} U_{v o}\left(t-t_{l}\right)$, takes the pattern

$$
\begin{gathered}
U_{V P C}^{t}\left(t-t_{i}\right)=A_{t} \int_{t_{t}-T}^{t_{1}+T} U_{v o}(\tau) \cdot U_{v o}\left(t-t_{i}-\tau\right) \cdot d \tau, \\
S_{V C}(f)=S_{V o}(f) \cdot S_{o c}(f) \cdot\left(m \cdot S_{v o}(f)\right)=m \cdot S_{o c}(f) \cdot S_{v o}^{2}(f) .
\end{gathered}
$$

This pattern coincides with that of an autocorrelation function of a sweepsignal. Correlation of vibrogram with the sweep-signal turns it into a vibro-pulse seismogram corresponding to pulse source generating short and powerful signal of an autocorrelation function form. As an autocorrelation function is symmetric, separate harmonic components have similar zero phase at $t=0$. It means that correlation has phased the sweep-signal frequency components. There has taken place time compression and change of amplitude while the signal's energy remained the same and the power accordingly increased.

If $U_{v o}(t)$ is presented, for example, by linear frequency modulated signal $A_{V O} \sin \left(\omega t^{2}\right)$, and the "compression" of vibration seismogram is realized by its convolution with the sweep-signal $\sin \left(\omega t^{2}\right)$, then the phase shift of harmonic components without change of their amplitudes is carried out, with signal's energy remaining the same and the power increasing according the compression. Thus, the amplitudes of vibro-pulse seismogram separate waves are equal to waves of pulse seismogram which corresponds to eradiation of the signal having the form of an autocorrelation sweep-signal function and energy equal to that of a sweep-signal.

An autocorrelation function possesses the following main features. The correlation function width is measured as the width of its envelope and equals to:

$$
\Delta t=2 /\left(f_{2}-f_{1}\right)
$$

where $f_{1}$ and $f_{2}$ - the upper and lower frequencies of sweep-signal. 
The envelope has a bell-like form and is filled by oscillations, the characteristic frequency of which is equal to mean frequency $\bar{f}=\left(f_{1}+f_{2}\right) / 2$.

An autocorrelation function pulse has the main maximum at $t=0$. Time between two negative extrema about the main maximum is $\Delta t=1 / 2 \bar{f}$

So, the choice of upper and lower frequencies determine the main autocorrelation function form parameters.

In real conditions the signal radiated by a seismic vibrator does not repeat the form of force $F(t)$. It is connected with the peculiarities of eradiation at small dimensions' source and medium inhomogeneity near the vibrator and complex interaction between vibrator and soil.

The problem of optimal signal forming is well studied in seismic prospecting. To solve this problem, a complicated form of vibrosignal frequency modulation, combination of several actions different in frequency and duration of eradiation are used. Such technique called combi-sweep provides ample opportunities for forming of vibro-pulse signal. The choice of bordering frequencies of a sweepsignal is limited by strong absorption and scattering of short-period waves from the side of upper frequencies, and from the side of low frequencies it is limited by high seismic impendans of medium. In the solution of the problem of determination of underground nuclear explosion yield it is necessary to determine the frequency characteristic of the medium from the source towards the receiver. To do it, it is advisable to use the sweep-signals having approximately the same frequency characteristic in the registration point as that of the UNE's seismic signal. The strict similarity of registered wave form from the underground nuclear explosion and the pulse form wave from the vibrator is not necessary.

\subsection{Frequency characteristic of the medium}

In a simple case when dealing with linear system its frequency characteristic is determined as ratio of signal's spectrum at output to its spectrum at input. The case is somewhat more complicated when the transformation of signal passing through the medium is studied. The signal at mediums entry is a complicated function dependent on amplitude, angle of departure of a seismic ray and frequency. Multi-path spreading of a seismic wave, the existence of different types of waves - longitudinal, transversal, surface, -all this raise a most complicated distortion of the entrance signal and consequently an extreme spatial changeability of frequency characteristic.

The idea of method is described above in the item 3 . It is possible to determine medium's frequency characteristic by a single wave - the first longitudinal, transversal, surface one. It considerably simplifies the problem and allows to apply comparatively simple and effective method.

Let us consider the method of medium frequency characteristic determination by the first longitudinal wave $P$. 
Suppose that the source $O$ radiates the wave $P$ having the form of a prolonged sweep-signal $P_{v o}(t)$, its spectrum $S_{v o}(f)$; here and below we consider only the amplitude spectrum. The signal when passing as a longitudinal wave by the short test time route to the registration point $C$ has form $P_{v C}(t)$, its spectrum $S_{v c}(f)$.

The P-wave signal has large duration and interferes with other signals which arrived to the observation point $C$. But the vibro-pulse seismogram obtained as a result of correlational compression does have the normal form, the longitudinal wave $P_{V C}(t)$ is distinctly resolved.

The spectrum of $P_{v C}(t)$ is $S_{V C}(f)$, it is the product of three factors:

$$
S_{V C}(f)=S_{V o}(f) \cdot S_{o c}(f) \cdot\left(m \cdot S_{V o}(f)\right)=m \cdot S_{o c}(f) \cdot S_{V o}^{2}(f) \text {, }
$$

where $S_{o c}(f)$-is the medium's amplitude frequency characteristic, $S_{v o}(f) \cdot S_{o c}(f)$ is the spectrum of noncorrelated P-wave which arrives to the registration point $\mathrm{C}$. The non-dimentional function $m \cdot S_{v o}(f)$ is the spectrum of the signal used for correlational compression, $m$ is the constant coefficient which selection determines the amplitude level of vibro-pulse seismogram.

The medium's amplitude-frequency characteristic is

$$
S_{o c}(f)=m^{-1} \cdot S_{v c}(f) / S_{V o}^{2}(f) \text {. }
$$

Spectra forming the right part of this equality are known from experiments. $S_{v c}(f)$ is the spectrum of P-wave registered on vibro-pulse seismogram, $S_{v o}(f)$ is the P-wave spectrum registered near the source.

The P-wave form near the source may be determined in different ways.

In one mode the value of force upon the vibrosource border and the soil vibration velocity in the point of force application is used. Another mode may be based on the $P$ near source characteristic męasurement. Both modes will be looked upon below.

Errors of medium's frequency characteristic determination are connected with the following main sources:

- influence of microseismic noise upon the determination of vibro-pulse wave $P$ spectrum,

- influence of wave $P$ interference with scattered and other secondary waves arriving with small delay, that is with signal generated noise.

- error in wave $P$ spectrum determination near the seismic vibrator.

The methods of decreasing the microseismic noise are known. They are the signal's intensity increase, different ways of noise suppress: frequency filtering, polarization filtering, seismic arrays usage, and in particular micro-arrays. 
The influence of wave $P$ interference with secondary waves could not be decreased by increase of source power and wave $P$ intensity because in such case there will take place proportional increase of intensity of signal generated noise.

In other respects there may be used the same methods that in the previous case - the use of micro-arrays, different modes of filtering. Methods of signal's "compression" allowing to get a more short signal than an autocorrelation function are of particular importance in this noise fighting. Specifically, one of such methods uses the resolution of seismogram upon functions which have the form of $P$ wave (Magnitskaya,N.,N., 1981). This method has good reserve of development, its application will allow to transform the vibro-seismogram in such a way that it will take the look mostly corresponding to underground nuclear explosion seismogram.

Errors in determination of longitudinal wave $P$ form and amplitude near the source are bound with some reasons, the main of which are: the influence of medium's seismic properties heterogeneities near vibrator, longitudinal and transversal wave interference, non-linear distortions of a signal. Heterogeneities of seismic properties influence the characteristic of seismic eradiation direction. This factor influence may be theoretically calculated if there are data upon the structure and properties of the environment. Especially significant may be the influence of layering.

Seismic eradiation power $J$ is determined by the following formula (Clymer, et al.,1977)

$$
J=F \stackrel{\circ}{U} \cos \phi
$$

where $F$-pick force, $\stackrel{\circ}{U}$ - particle velocity beneath the platform of vibrator, $\phi$ - is the phase shift between $F$ and $\stackrel{\circ}{U}$.

If the medium is stratified than particle velocity depends greatly upon the position of source with respect to seismic borders and depends on oscillations frequency. Methods of medium layering control upon the eradiation character are well elaborated and may be applied at calculations of vibrator's power characteristics and wave $P$ spectrum form far off the source.

Study of lateral inhomogeneities near the. source and its influence on eradiation is much more complicated and special methods of seismic tomography investigations are needed particularly near borehole tomography.

Longitudinal waves $P$ and transversal waves $S$ interference is observed in comparatively small range of distances from the source, from approximately 0.1 of minimal wave $S$ length up to length of $P$-wave signal. The observations in the satellite borehole should be based on these figures. In the nearing zone it is expedient to control the $P$-wave form on the base of previously given formula for eradiation yield. Outside the interference zone, $P$ and $S$ wave are resolved on vibro-pulse seismogram.

Borehole observations for the control of vibrator's eradiation yield and longitudinal wave-form could give a more objective and precise information than 
observations under vibrator's platform. When using surface vibrator the borehole may be situated near the platform. In this case seismic measurements would characterize the downward eradiation of a vibrator. The angles of departure of seismic eradiation being small, about $20^{\circ}$, and the direction characteristic of vertical force at such angles are nearly maximum, the evaluation of vibrator's eradiation power is enough objective. Taking into account that transversal wave eradiation yield downwards could be neglected when compared to longitudinal wave eradiation yield, the bore-hole depth may be taken proceeding not from the above mentioned conditions of longitudinal and transversal waves separation but from conditions of longitudinal wave observations in a "remote zone", which is not less than one half of a most long wave length. If the most low eradiation frequency is equal to $1 \mathrm{~Hz}$, longitudinal wave velocity is $3500 \mathrm{~km} / \mathrm{sec}$, than the seismic observations bore-hole depth should be about $1200 \mathrm{~m}$. That can be also recommended while using mine vibrator in a nuclear explosion hypocenter. Borehole - satellite may be near the mine. The study of eradiation direction dependence upon the angle of departure requires the borehole for seismic observations to be situated at distance not less than one half of maximum length of the wave from the hypocenter. Its depth should be conditioned by a seismic ray corresponding to maximum registration distance, transmitting through the borehole. With previous example conditions, the hypocenter depth equal to $500 \mathrm{~m}$, minimum angle of departure $20^{\circ}$, distance from the hypocenter to the borehole $1200 \mathrm{~m}$, the borehole's depth must be 3300 $\mathrm{m}$. Thus, the investigation of eradiation direction calls for rather large expenses on bore-hole observations. The expediency of such observations can be evaluated only experimentally.

Nonlinear distortions of signal form are controlled on instruments record situated in the zone of considerably large deformation. For seismic vibrator of about 100 ton power with coefficient of medium's nonlinearity

$K=\rho V_{p}\left(d V_{p} / d p\right)=10^{4}$

$\rho$ - density, $V_{p}$ - longitudinal wave velocity, $p$ - stress and the frequency of

$100 \mathrm{~Hz}$ this zone reaches the distance of about $1 \mathrm{~km}$ from the source, with the level of nonlinear distortion on the zone's border up to $10-30 \%$.

Significant part of power is concentrated in upper harmonics, the propagation of which is bound with relatively strong absorption and scattering. These harmonics are greatly extinguished on the way of spreading towards the seismic station and the rest part of them represents some kind of noise signal that will be rejected at correlational compression of seismogram.

The sources of calibration errors may also be the peculiarities of wave excitation by nuclear explosion and their propagation, which could not be predicted on base of investigation of wave field from the vibro-sources. These peculiarities are bound with rocks' tectonic energy release followed by seismic 
waves generation. and difference in nonlinear seismic effects taking place at propagation of quasiharmonic and pulse signals.

Release of tectonic energy by explosion is difficult to evaluate, though in experts' opinion in some exclusive cases it is commensurable with UNE's seismic energy, which leads to calibration error up to 0.3-0.5 magnitude's unit. Application of seismic signals' spreading paths vibroseismic sounding will give the opportunity to estimate the part of tectonic energy released by an explosion.

If an explosion do not release tectonic energy, then the amplitudes' ratio of vibrator and explosion seismic waves registered on seismic stations should be approximately equal.

Tectonic components presence in a seismic wave field should tell in spatial variation of explosion and vibrator aroused waves amplitudes' ratio. This is bound with anizotropy of tectonic earthquake focus eradiation acting simultaneously with an explosion.

Tectonic component of seismic eradiation may be quantitatively evaluated on the base of data on spatial variations of seismic waves aroused by vibrator and explosion amplitudes' ratio. Naturally, there must be used not only longitudinal but also transversal and other types of waves.

Released tectonic energy should be bound with explosion yield, aftershock activity of source area. Possible initiation of seismoacoustic emission by vibrator near the epicenter indicated to the existence of large tectonic stresses and to the possibility of tectonic energy release by nuclear explosion.

Longitudinal pulse seismic wave spreading from the source towards registration point is expressed in solitone features manifestation. According to observation data in teleseismic zone the width of $P$-wave impulse at level 0.5 from maximum has decreasing tendency at UNE yield increase (Gamburtzeva,N.G.et al.,1987). Consequently $P$-wave spectrum should be relatively stable. In particular this tells in relatively weak extinction of its short period part. It is evident that frequency characteristic of medium determined according to data on UNE's wave spreading should therefore differ from frequency characteristic defined by vibrational sounding of the Earth. The question of role of seismic signals nonlinear distortions spreading at large distances still remains practically unstudied. And so the investigation of nonlinear processes at seismic waves of different types spreading in crust and mantle must be one of the essential parts of the program of creation of UNE calibration methods with the help of vibrators.

So, the role of errors in determination of medium frequency characteristic and calibration of UNE is bound with medium features inhomogeneity and stress state near the source. In every concrete case depending on experiment set up there separate evaluations should be done. That should be achieved by combination of in situ observations. These methods development needs conducting of special theoretical and experimental researches. 


\subsection{Discussion, Conclusion}

UNE's calibration with the help of vibrators depending on methods applied might have different degree of precision and effectivity:

- detailed investigation with the help of sounding of the wave-track from the hypocenter or UNE's epicenter towards the seismic stations;

- less detailed investigation, estimation of geological conditions of the source region and the region of seismic station location upon the wave field.

Detailed study of seismic wave propagation from the source to the seismic station allows to determine medium frequency characteristic for P-wave and to use this characteristic for UNE calibration.

The above considered possible calibration errors may have different nature and under circumstances may exert different influence upon the precision of UNE's yield determination.

Now, it is not the errors we shall not be able to correct that is important for us, but to what extent will improve the UNE's yield determination when calibrated with the help of seismic vibrator.

The investigation of vibrosignal passing from the source to the registration point will allow to practically completely eliminate errors bound with geometrical spreading of seismic wave, its absorption and scattering. These factors cteate spatial variations of seismic waves amplitude, having different scales and contrast due to the character of crust and mantle inhomogeneities, oscillation frequency. As a result of variations UNE's yield is determined with an error, the uncertainty coefficient of which is evaluated as $3.5-8$. In this summary evaluation the influence of test site conditions, propagation track and conditions of registration is roughly equal and is characterized by 1.5-2 uncertainty coefficient. This value corresponds to a scatter of data bound with choice of concrete place of UNE conducting within the test site.

Thus, the direct calibration of UNE's yield may improve the precision of yield determination by approximately 1.1-1.2 even on well studied test sites with the use of calibrated seismic stations'nets.

Less detailed investigations presuppose the attestation of new seismic stations, regions and site of UNE's possible conducting. Such problem is usually solved with the help of regional distant earthquakes. Usage of the earthquakes' records from different azimuth in ratio to seismic station allows to determine corrections of amplitude and arrival time in azimuth and epicentral distance function for this very station. The drawbacks of this method are its relatively low calibration precision and grearer duration of observations. Epicentral zones calibration is carried out by observation of earthquakes by seismic network. So far the aseismic regions turn out to be unstudied.

The solution of the problem demands the application of artificial sources, explosions and vibrators. Calibration explosions should have large enough yield of about $0.5 \mathrm{kt}$. It creates not only technical but also ecological problem which greatly limits the possibility of the explosions application. The use of vibrators for 
direct and inverse calibration of seismic stations regions and regions of possible UNE's conduction is bound only with technical difficulty.

The application of the following calibration schems is possible: direct calibration of supposed test site; a vibrator performs eradiation seances in several points, receiving is performed by stationary and temporal seismic stations.

Inverse calibration of the test site: temporal seismic stations are set on the supposed place of UNE's conduction, the point of vibrator's setting are chosen in different azimuths and on different distances from the test site. The seismic reciprosity principle is used in determination of amplitude corrections for the test site. This principle betoken that the replacement of a source by a vibrator and vice versa with their sensitivity axes' orientation being preserved, the wave field character also preserves, strict control of reciprocity principle by ultrasonic modelling and by experiments in situ confirms its truth.

Reversed calibration of seismic station may be performed by a vibrator set near the station. That will provide the opportunity to study the propagation of a signal from this very station to another registration points situated in different azimuths and at different distances from the investigated point.

It seems that, when planning the calibration system of certain regions of the supposed UNE's conduction and registration, there may be applied a combined system using both direct and inverse observations scheme. Data on direct observations which are, possibly, more rare may be interpolated by area with the use of a more dence system of inverse observations.

The described approaches to the solution of UNE's calibration problem still have the character of recommendations for corresponding research and development works conduction. Vibrator have not yet been used for UNE's direct calibration, so now we have only one possibility - to synthesize the proposed method from separate parts more or less studied in different branches of geophysics.

There are many things still unknown in this branch of geophysics and further explorations are necessary. Anyhow, the outline of the method is already seen. The following conclusions are to a great extent due to the held investigation and to a certain extent they reflect the opinion of geophysicists engaged both in vibro-sounding of the Earth and UNE's calibration problems.

1. On the basis of powerful seismic vibrators and "Vibroseis" method there may be created a method of UNE's yield calibration.

2. Creation of powerful vibrators and tools for their exploiting will need considerable expences. But it seems to be the only way of development of nuclear explosions calibration method, and as a matter of fact, of the whole globe calibration, which is an important stage of investigations bound with the threshold reduction and with total prohibition of nuclear weapon tests. So, in the total, vast expences would be justified by creation of calibration method of high precision and universality.

3. It's reasonable to start investigations from elaboration of chemical explosions calibration technique on the existing vibrotest sites, from calibration 
of several UNE already held in Russia and USA with the help of some experimental models of powerful seismic vibrators.

In these studies it is advisable to use mobile stations PASSCAL. It is also expedient to make 3-5 experimental models of mobile 50 ton seismic vibrators. This may be done by modification of already existing 30 ton Gomel vibrators. The question has been practically solved in 1989, but for the lack of financing for the elaboration. 


\section{Conclusion}

The idea of UNE's yield calibration with the help of seismic vibrators is simple enough, though its accomplishment is bound with solution of complicated technical and scientific problems: creation of new generation of powerful seismic vibrators, elaboration of medium frequency characteristics methods and elaboration of UNE's yield direct calibration methods on thier basis. Some parts of this large-scale problem are already being solved by geophysicists: a "Vibroseis" method, applied in seismic prospecting appeared to be the basis for works on "Vibrosounding of the Earth" program; there are prototypes of powerful seismic vibrators and experience of lithosphere vibrosounding, registration of signals at more than $300 \mathrm{~km}$ distances and detection at $2500 \mathrm{~km}$ distances. This experience is the base for elaboration of technique and methods of UNE's calibration with the help of powerful vibrators.

Evaluation of seismic energy necessary for calibration show that while designing the experiments one should orientate at $5 \cdot 10^{9}$ Joule value as an equivalent of seismic eradiation corresponding to UNE of $1 \mathrm{kt}$ yield. Vibrational eradiation energy for UNE calibration by seismic wave registration at distances up to $1000 \mathrm{~km}$ from the source may be less or even more less than this value which depends on circumstances.

Seismic vibrator must produce enormous force of hundreds and even thousands tons to radiate such amount of energy in $1-5 \mathrm{~Hz}$ frequency band during several hours. Instead of creating huge machines it is expedient to apply modulus-principle and use a battery of 50-100 ton moduli instead of one giant vibrator. The necessity of mine vibrators usage for seismic waves eradiation from UNE's hypocenter should be specially studied. Apparently, in many cases it will be enough to use surface vibrator set in UNE's epicenter.

Effectivity of UNE's yield direct calibration with the help of vibrosources to a great extent depends on whether UNE is held on a well known test site, e.g. Semipalatinsk or Nevada ones, and is registered by well calibrated station, or an explosion is held for the first time in a new place and is registered by uncalibrated stations. In the first case the direct calibration will allow to improve the precision of good evaluation and to diminish the uncertainity coefficient of yield determination error, for example from 1,2 to 1.1 . In the second case the 1.1 value might also be achieved, but with apriori uncertainty coefficient 3 or more.

Calibration of not yet functioning test sites, territories on which UNE's could be held in principle, calibration of new seismic stations is probably a more actual problem than defining the yield of explosions held on existing test sites. Application of seismic vibrators seems to be the only way of its quick and effective solution.

Scientific part of the program of UNE calibration by vibrators should be aimed at solution of the already known but not yet solved problems: investigation of nonlinear effects arousing in a near field of a vibrator and on a long tracks of 
vibrosignals propagation; investigation of solitone properties of longitudinal wave, generated by UNE. The problem of creation of low frequency parametric sources on the base of high frequency vibrators is of special interest in view of nonlinear effects investigation. It is possible that this very type of sources would turn out to be most perspective for practical application.

Another important problem is a nuclear explosion tectonic energy release on the base of vibrator and explosion wave field structure comparison. To solve it, it is reasonable to held special vibrator calibration of those UNE during which vast amount of tectonic energy has been released.

The beginning of investigations on UNE vibrator calibration problem may be organized on the base of existing vibro test sites Bystrovka (Novosibirsk region) and Uznoz (Gomel region, Belorussia). It is necessary to study the process of seismic waves eradiation by powerful vibrators, to held a detailed investigation of seismic wave field structure at distances up to $500-1000 \mathrm{~km}$; to carry out experiments on chemical explosions calibration, PASSCAL stations are advisable to be used in these works.

This investigations may be carried out by Russian engineers and geophysicists with participation of American colleagues. Expenses will be $50000000 \$$. The following organizations will take part in the work:

- Joint Institute of Physics of the Earth RAS;

- Computing Center of Siberian Branch of RAS;

- Joint Institute of Geology, Mineralogy and Geophysics of SB RAS;

- Institute of Geospheres dynamics of RAS;

- Special Design Bureau of JIPE RAS;

- Gomel Special Design Bureau "Seismofechnique"'Belorussia.

The next stage of work might be the calibration of explosions already held on Semipalatinsk and Nevada test sites. This problem's solution gets complicated because the soil in UNE's epicenter has become friable and a vibrator should be turned aside from the epicenter. To perform this investigation one might use the available prototypes or make new types of vibrators.

The further investigations plan will significantly depend on results obtained during the first two stages.

Grounding of vibrator usage for UNE's calibration possibility is schematic and is done on the base of relatively limited amount of data, the obtained estimations need correction applied to concrete observation conditions.

Nevertheless the outlines of method are clear enough, now there are all grounds for its elaboration.

Positive results if obtained will be the base for development of the calibration program of the whole Earth - "Global Calibration".

\section{Acknowledgments}

Dr. N.G.Gamburtseva and Prof. J.A.Urusovsky who has given a number of valuable advices on various parts of the work has participated in the work on parts 4 and 5. The Prof. O.V.Kedrov's, Prof. A.A.Spivak's and Prof. V.J.Jushkin's consultations were of great use. V.A.Nikolaev has rendered much support in 
scientific and technical editting and preparation of manuscript, M.G.Belyaeva translated the text into English. E.V.Kapitchnikov has composed the text and figures on a computer. Many thanks to all of them.

*This work was performed under the auspices of the U.S. Department of Energy by Lawrence Livermore National Laboratory under contract No. W-7405-Eng-48. 


\section{Refences}

Clymer,R.V., McEvilly,T.V., Nevsky,M.V., Nikolaev,A.V. Experimental estimation of seismic vibrator radiatory power.In Collection: "Problems of vibrosounding of the Earth. Moscow, Nauka, 1977, p. 80-85.

Gamburtseva,N.G., Nikolaev,A.V., Khavroshkin,O.B., Tsyplakov,V.V., Solitonic properties of teleseismic waves. Transactions of Acad. of Sc. of USSR, v.291, No4, 1986.(Russian).

Gustchin,V.V., Dokuchaev,V.P., Zaslavsky, Yu.Yu, Konjukhova,N.D. On power distribution betweel different types of radiated waves in semilimited elastic medium. In book: Investigation of the Earth by non-explosive seismic sources. Moscow, Nauka, 1981, p.113-118.(in Russian)

Isakovich,M.A., General acoustics. Moscow, Nauka, 1973, p. 470.(in Russian)

Jushin,V.J., Geza,N.J., Velensky,V.V., Mishurov,V.V., Speransky,N.F., Savenykh,V.S., Astafjev,B.A. Vibroseismic monitoring in Lake Baikal region. In book: New methods and tools for seismic observations. Moscow, 1994, p. 3851.(in Russian)

Kirichenko,V.V., Krajev,Yu.A., Peshkov,A.B., Pirsov,V.A., Stcherbakov,P.N. Application of seismic method in investigations of underground nuclear explosions yield at 1974 Threshold Test Ban Treaty control. International Conference "Geophysics and modern world. Moscow, 1993. P.29.(in Russian)

Magnitskaja,N.N. Efficiency of vibrosignals recording. In book: Investigation of the Earth by non-explosive seismic sources.Moscow, Nauka, 1981. p..204210. (in Russian)

Nikolaev,A.V. Seismic properties of friable media. Izvestija AN SSSR. Physic of the Earth, No2, 1967. p. 23-31.(in Russian)

Nikolaev,A.V. Seismic method in investigation of elastic waves velocities field variations. Izvestija AN SSSR. Physic of the Earth, No5,1970. p.103-111.(in Russian).

Nikolaev,A.V., Artjushkov,E.V., Chichinin,I.S., Galkin,I.N., Troitsky,P.A. Vibrosounding of the Earth. Moscow. VINITI. No2549-74. Dep. 1974.p.197.(in Russian)

Nikolaev,A.V., Galkin,I.N. (Editors in chief). Problems of vibrational sounding of the Earth. Moscow., Nauka, 1977. p.286. (in Russian)

Nikolaev,A.V., Galkin,I.N. (Editors in chief). Investigation of the Earth by nonexplosive seismic sources. Moscow. Nauka, 1981. p.336. (in Russian)

Nikolaev,A.V., Polycarpov,A.M. Method of seismic informativity determination of seismic station dislocation point. Copyright No 1183930, June,1. 1985. (in Russian) 
Peshkov,A.B., Kirichenko,V.V., Stcherbakov,P.N. Upon the possibility of earthquakes initiation by underground nuclear explosions. International Conference "Geophysics and modern world".Moscow, 1993.p.28-29.(in Russian).

Ryaboj,V.Z. Upper mantle structure of the USSR upon seismic prospecting data. Moscow, Nauka. 1979.p. 246.(in Russian)

Vasiljev,Yu.I., Vidmond,N.A., Molotova,Z.V., Nikolaev,A.V., Khavroshkin,O.B., Tsyplakov,V.V., Stcherbo,M.S. Experimental investigation of seismic radiation forces in a soft ground. Izvestija AN SSSR. Physic of the Earth. No1, 1986.p.5256. (in Russian) 


\section{Figures caption}

Fig.1. Scheme of UNE calibration. Seismic vibrator is installed in point $O$ on the shaft bottom. It radiates the sweep-signal which is received in point $\mathrm{C}$. Spectral characteristic of OC trace Svc/Svo is used for determination of source spectrum Sno of UNE which is explosed in point $O$

Fig.2. Seismogram of $40 \mathrm{~min}$ sweep-signal, $6-9 \mathrm{~Hz}, 100$ ton vibrator (a), source-receiver distance $300 \mathrm{~km}$; seismogram of 4 ton quarry explosion, distance $240 \mathrm{~km}$ (b)

Fig.3 Map of vibromonitoring experiment set up. 100 ton vibrator is installed in Babushkin, seismic station in Kharat. Distance $125 \mathrm{~km}$

Fig.4. Seismogram of vibromonitoring of Baikal Rift zone. 20-min sweepsignals registered in Kharat (a, b.c, d); $d$ - stacking of 24 sweeps

Fig.5. Scheme of seismic rays and hodograph $t$ (delta)

Fig.6. Empirical curve of P-wave particle velocity amplitude of $1 \mathrm{kt}$ UNE versus epicentral distance (a) and $P$ wave dominant period (b)

Fig.7. Scheme of DSS profiles and Semipalatinsk test site

Fig.8. Seismic shaft vibrators a - vertical vibrator with inertial mass; b, c centrifugal vibrator

Fig.9. Novosibirsk's 100 ton vibrator with two rotating masses. 1 - electric engins, 2 - iron piles and 3 - tank with sand press the platform to the surface. The rotating masses are covered by iron boxes 4 .

Fig.10. Novosibirsk's hydraulic vibrators.

1 - vertical 100 ton vibrator

2 - horizontal 250 ton vibrator

Fig.11. Gomel's mobile Vibrator.

Fig.12. Gomel's 100 ton hydraulic vibrator.

$$
\begin{aligned}
& 1 \text { - cylinder, } \\
& 2 \text { - platform, } \\
& 3 \text { - inertial mass, } \\
& 4 \text { - diesel engine. } \\
& \text { Boy } 140 \mathrm{sm} \text { tall }
\end{aligned}
$$

Fig.13. "Vibroseis" method. Seismic vibrator radiates the sweep-signal which is reflected from the boundaries I, II, III; $b$ - the single reflected waves, $c$ interference of reflections, $d$ - vibro-pulse seismogram obtained as a result of correlation of $C$ and a signals; $t 1, t 2, t 3$ - arrival time of reflections 

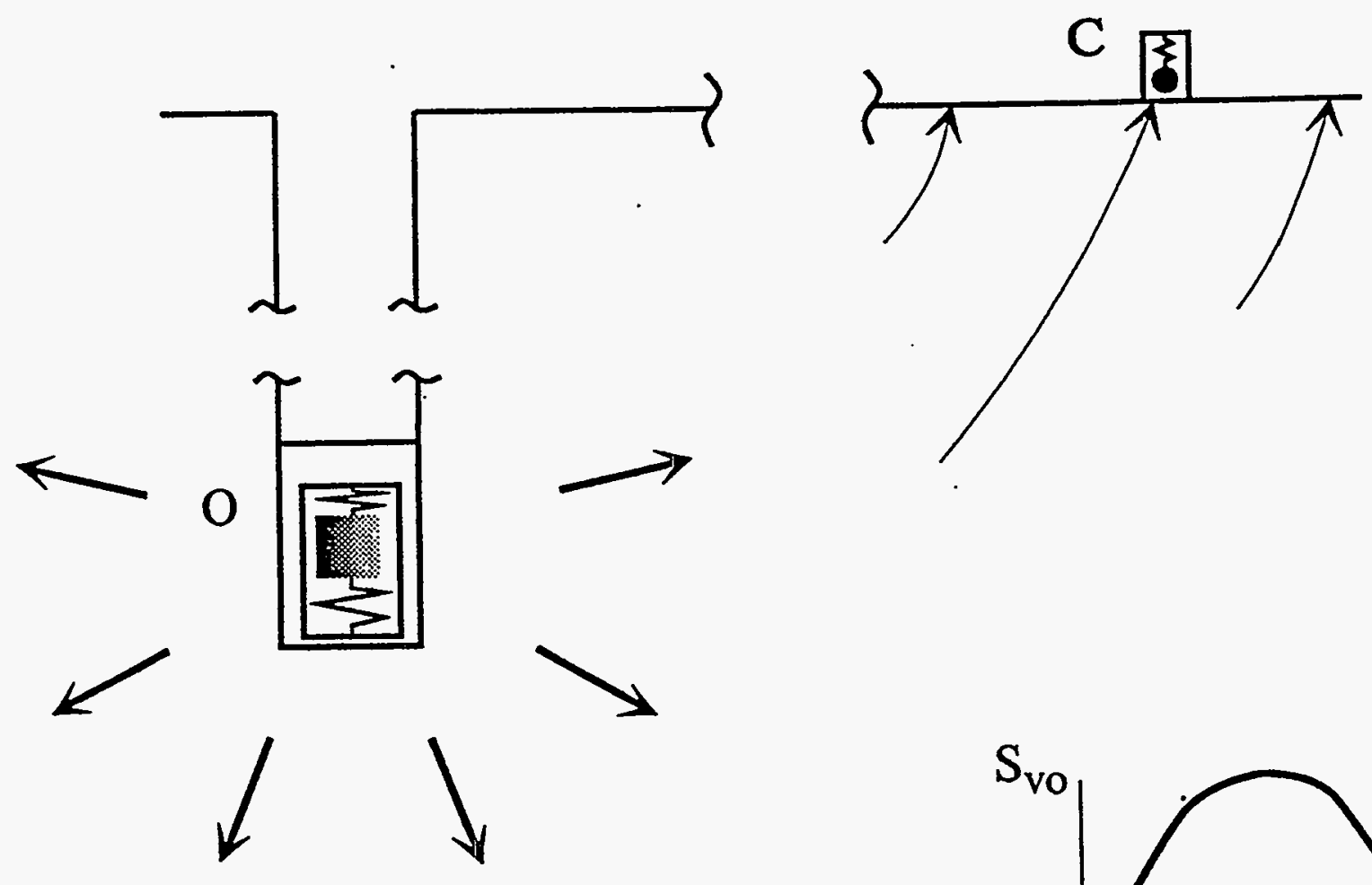

Sweep signal

HOMON
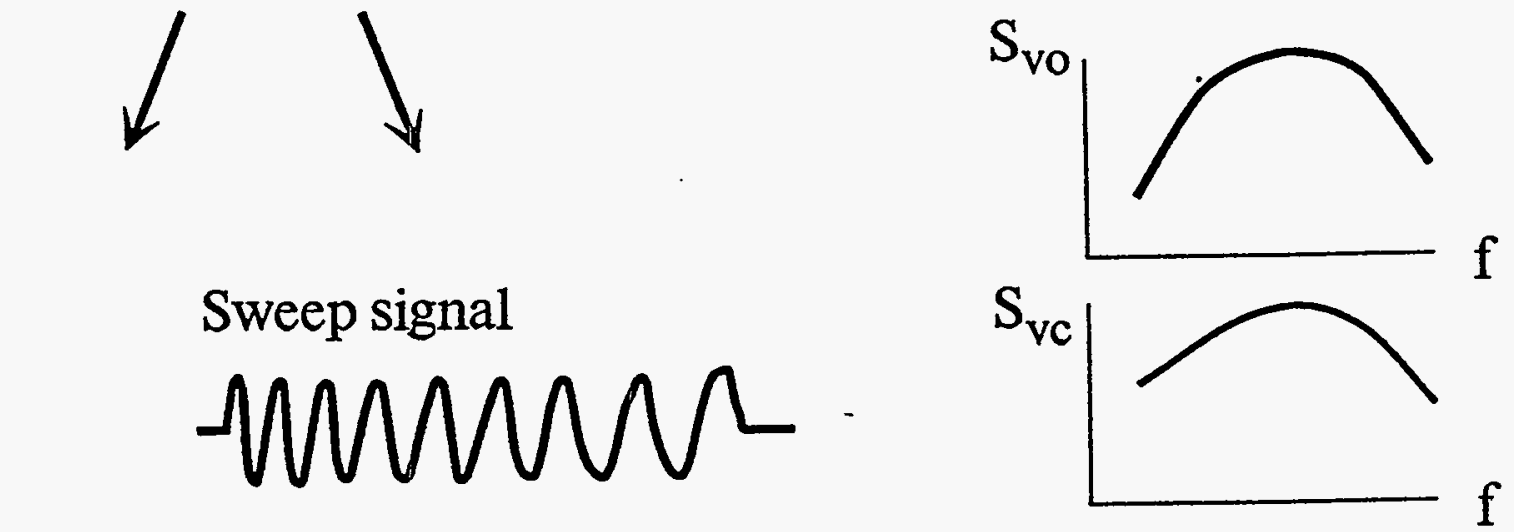

$\mathrm{P}_{\mathrm{vc}}(\mathrm{t}) \stackrel{\text { Vibrator }}{\sim}$

$\mathrm{S}_{\mathrm{vc}} / \mathrm{S}_{\mathrm{vo}}$

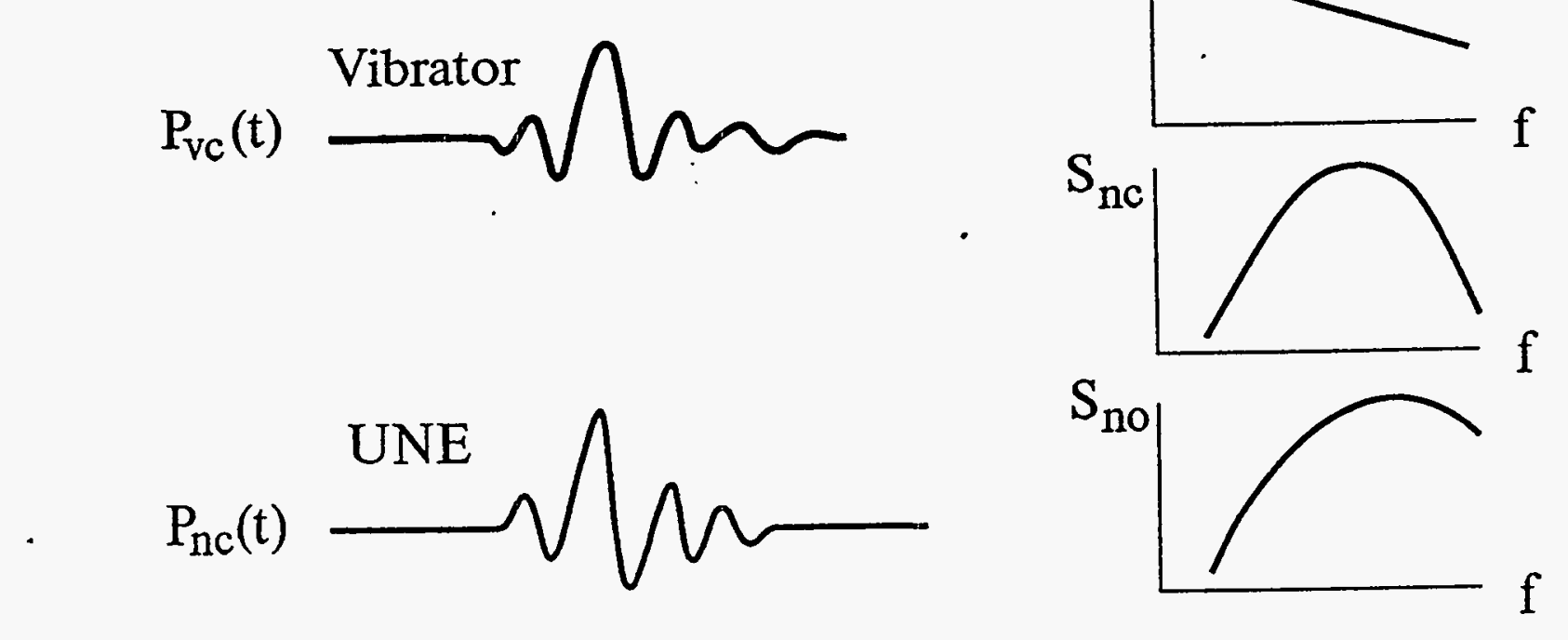

Fig. 1 


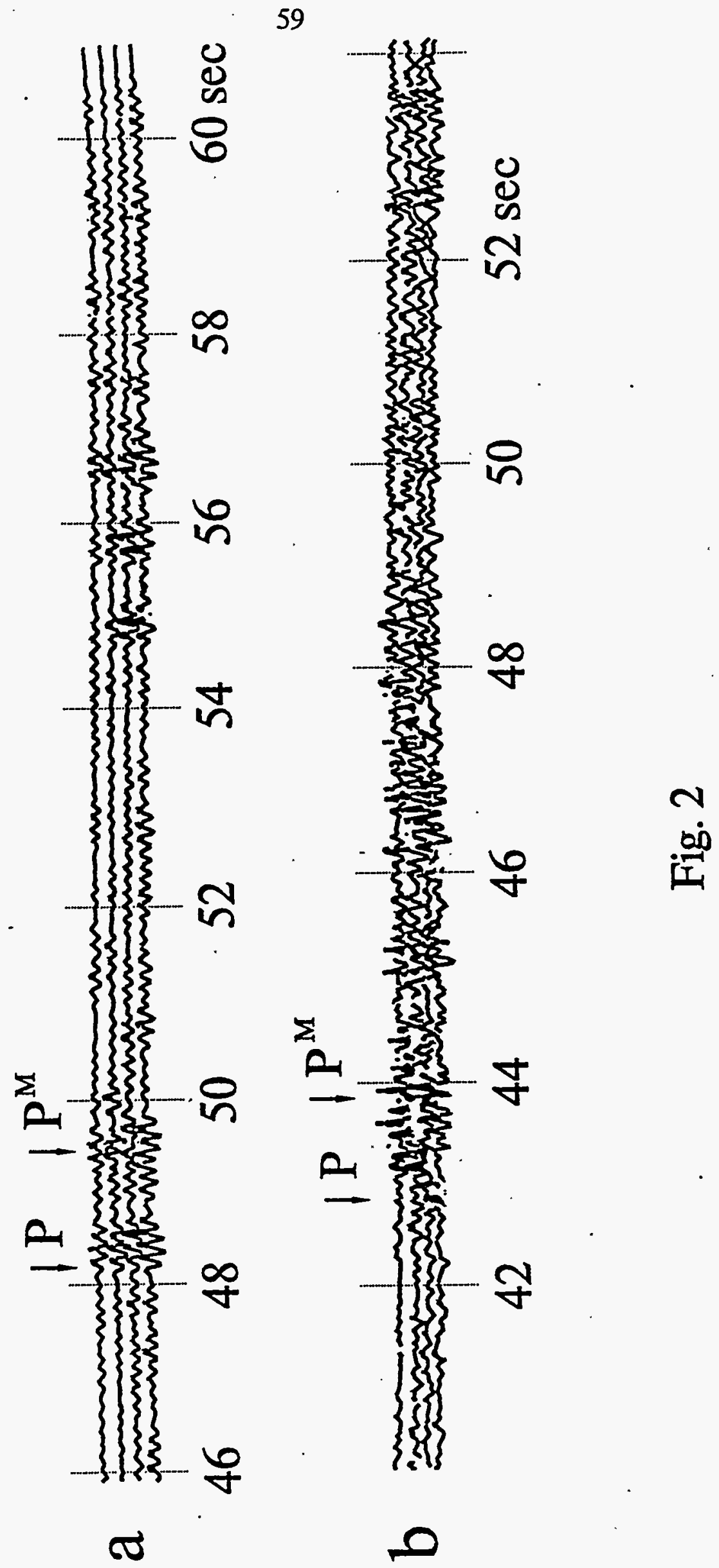




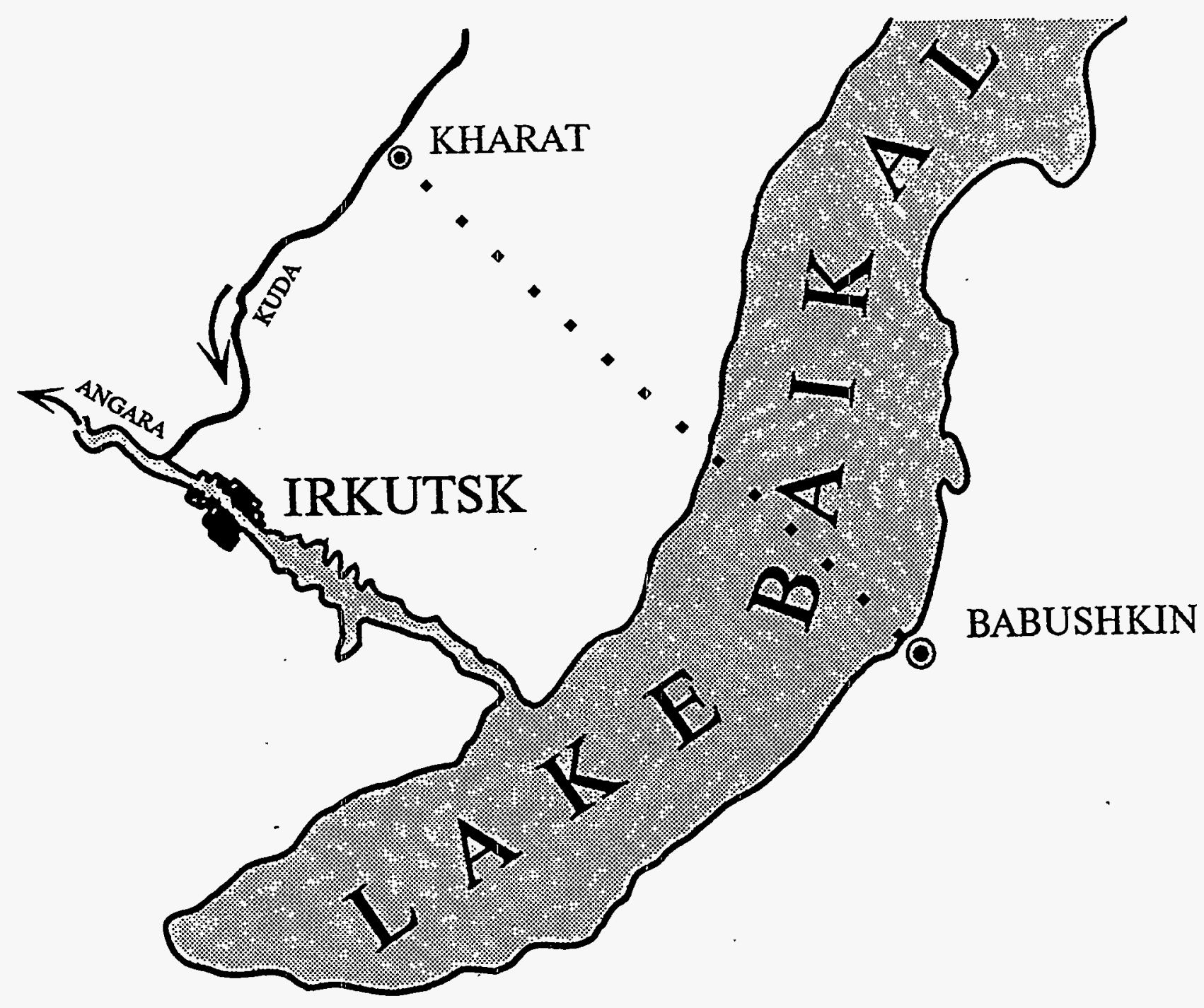

Fig. 3 


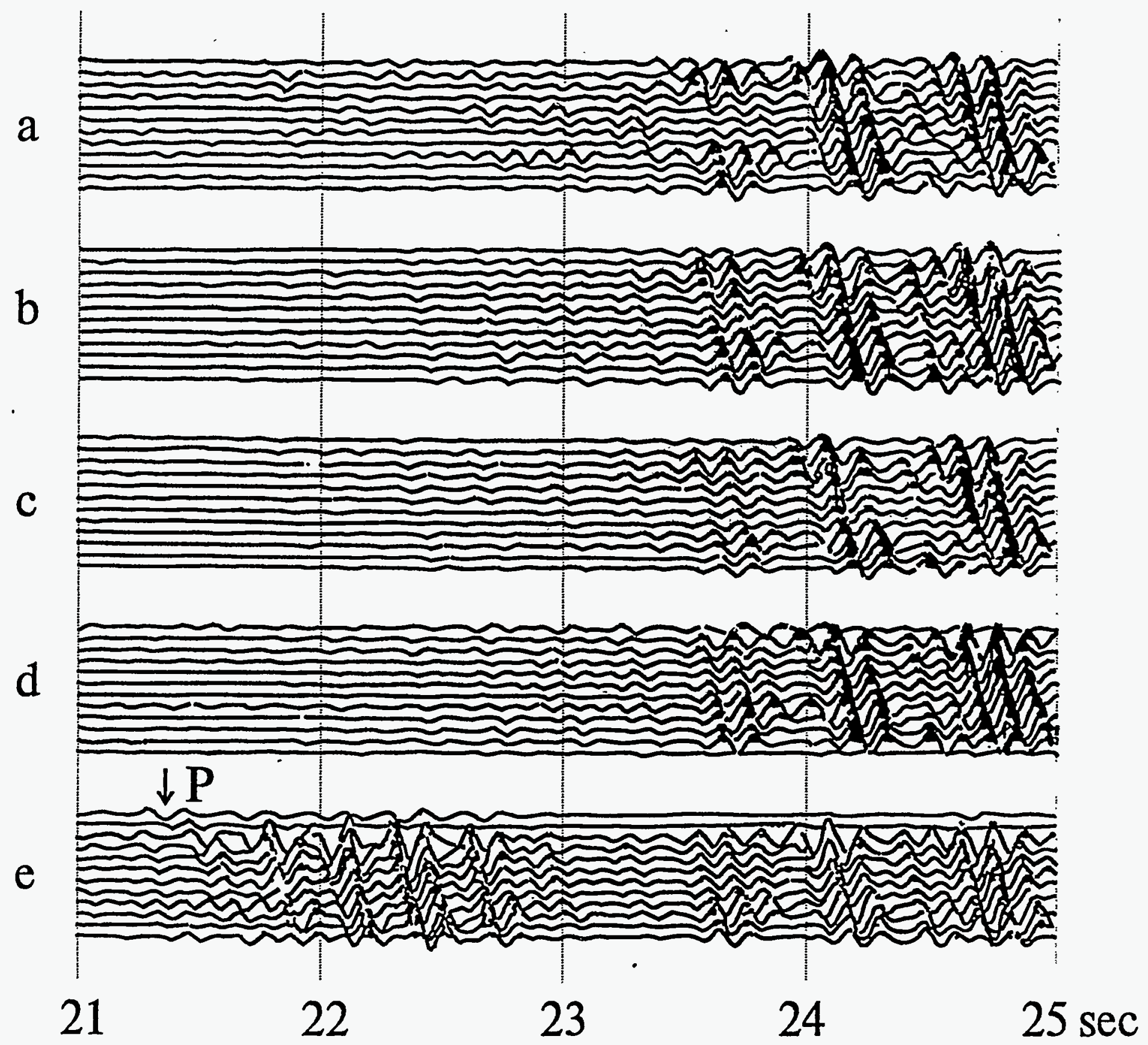

Fig. 4 


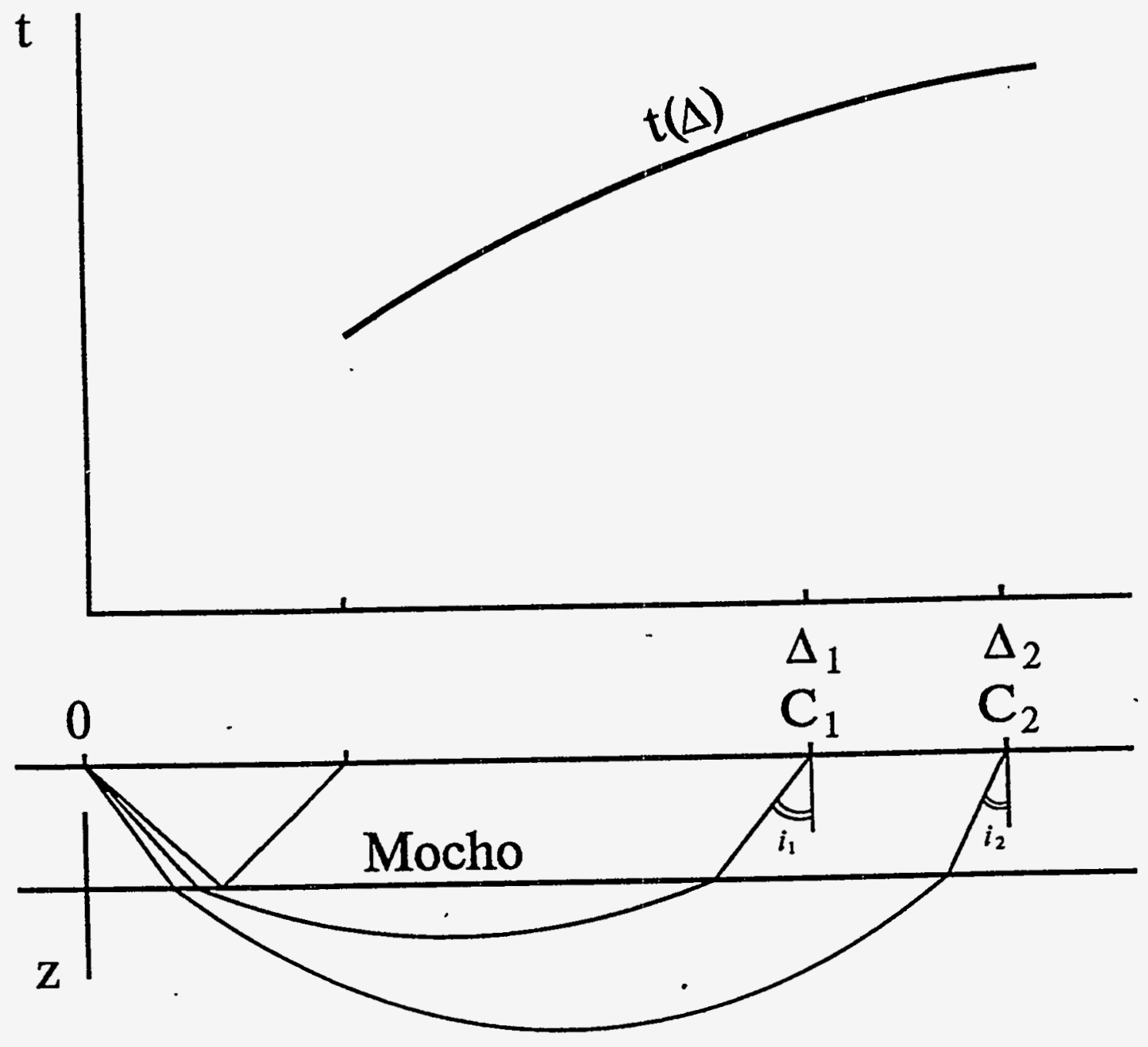

Fig. 5 
$\dot{\mathrm{U}}, 10^{-6} \mathrm{~m} / \mathrm{sec}$

a

a

b

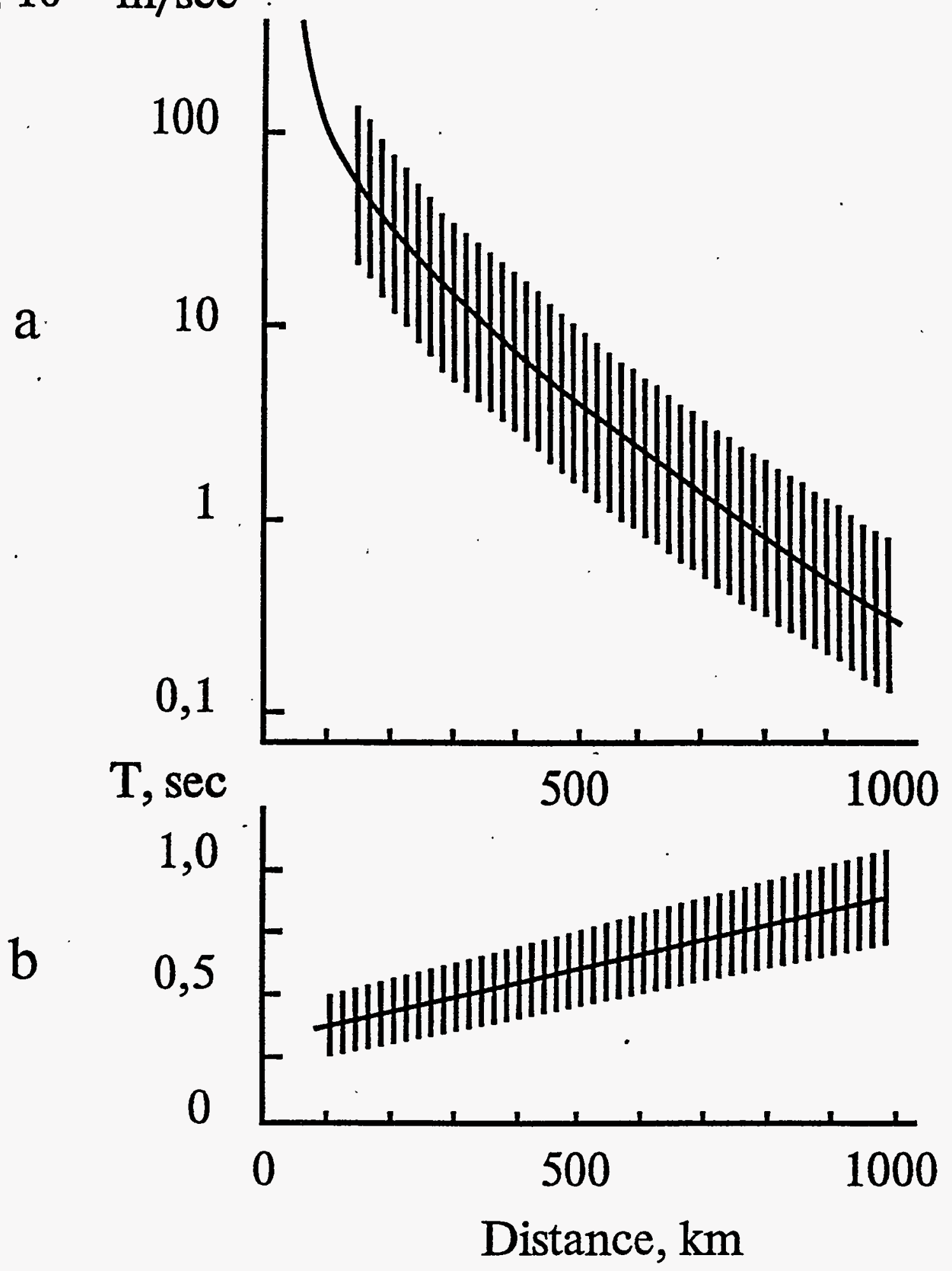

Fig. 6 


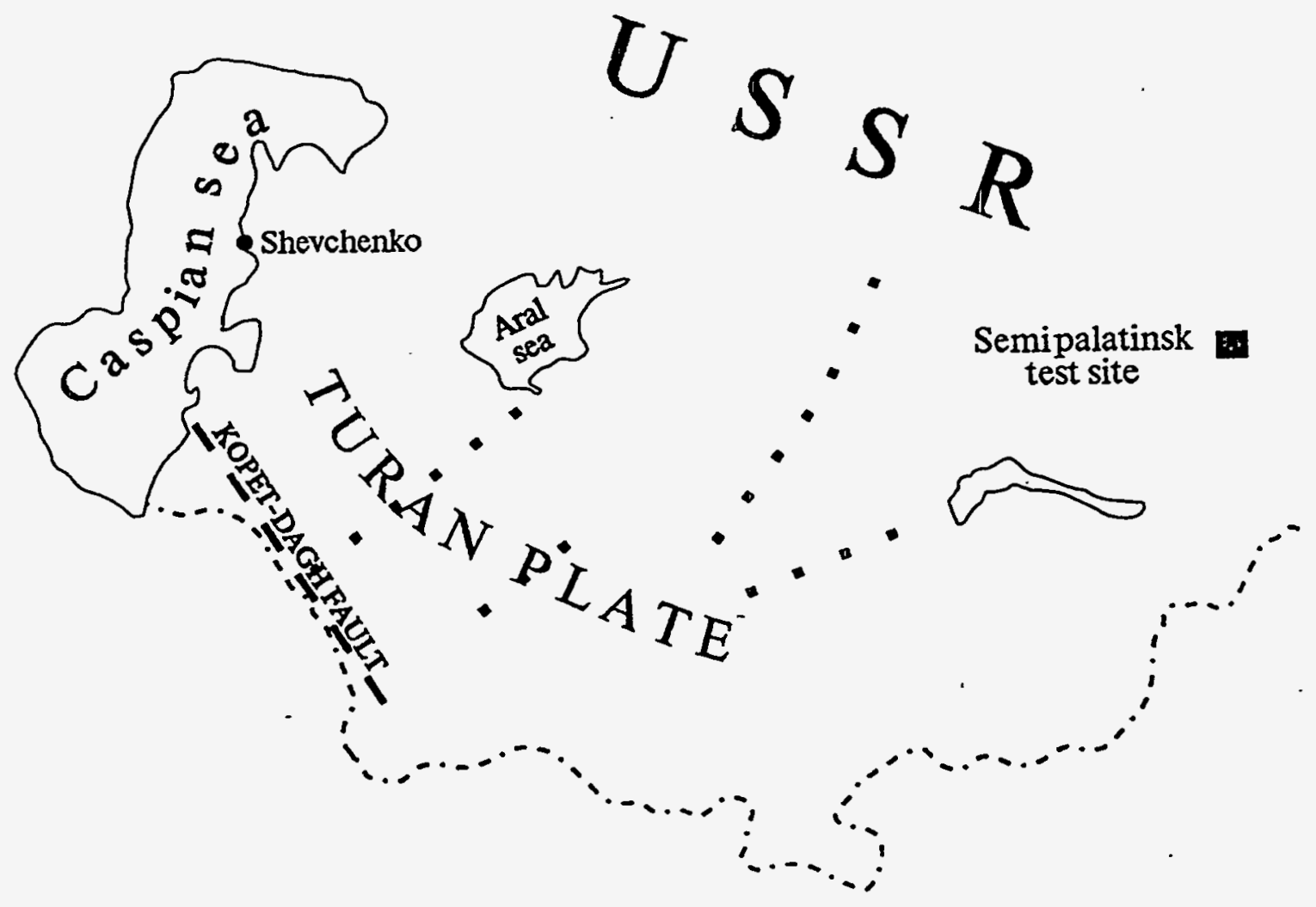

... DSS profile

Fig. 7 

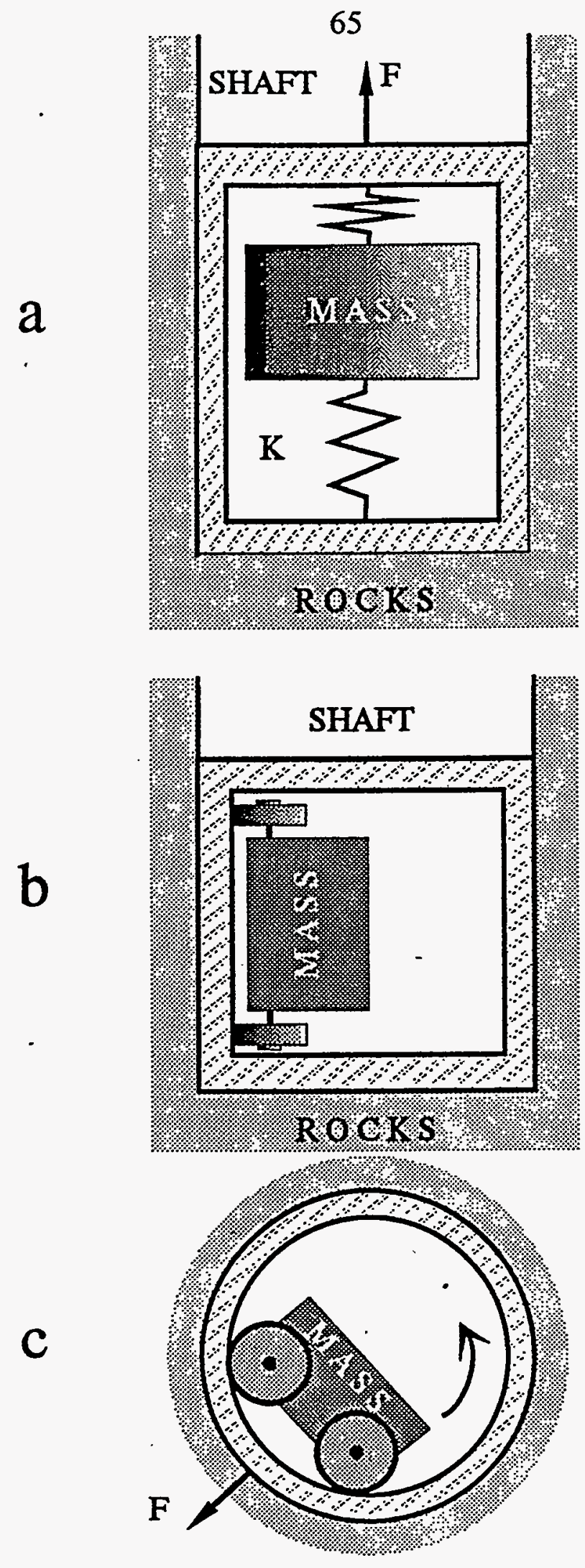

Fig. 8 


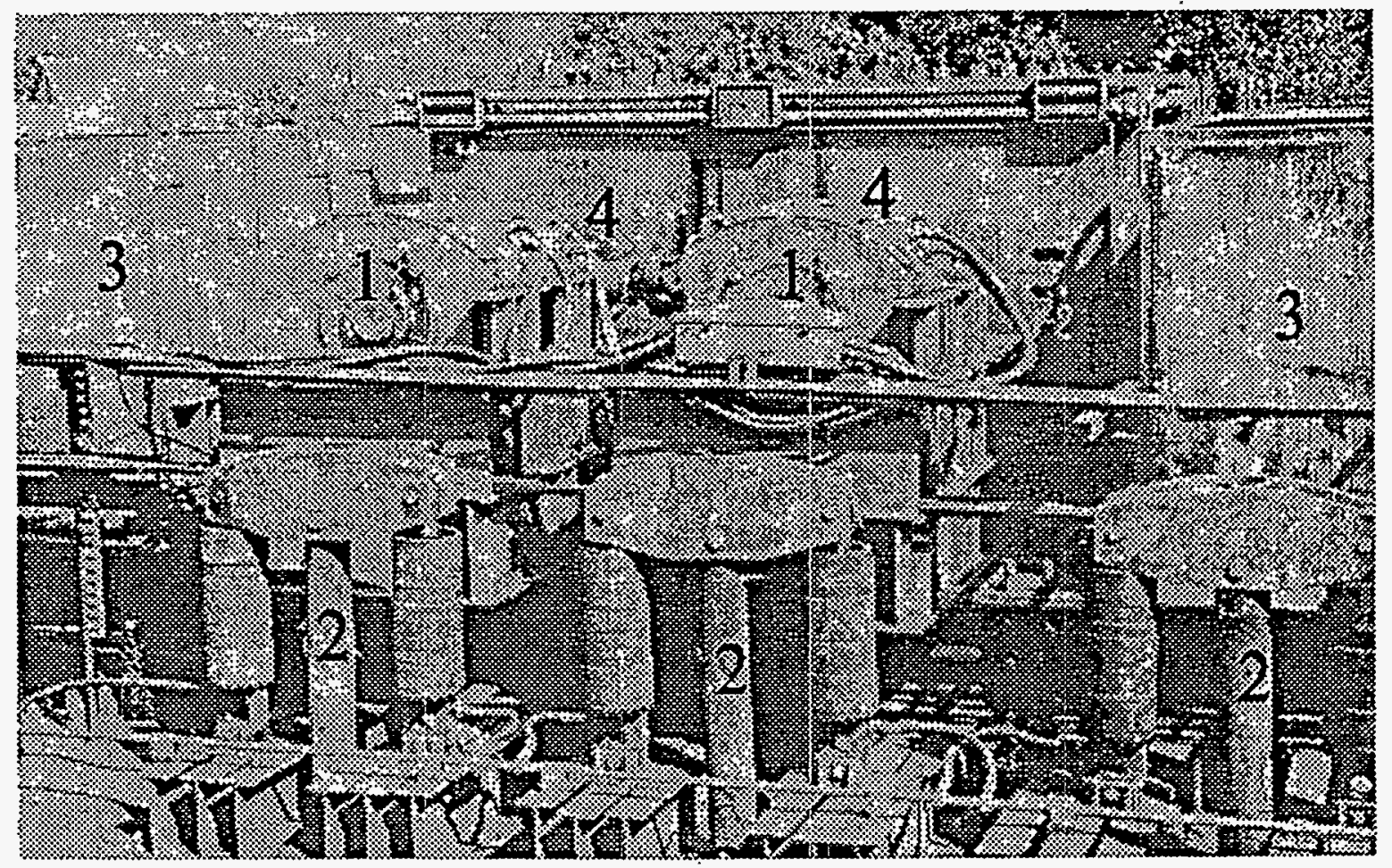

Fig. 9 


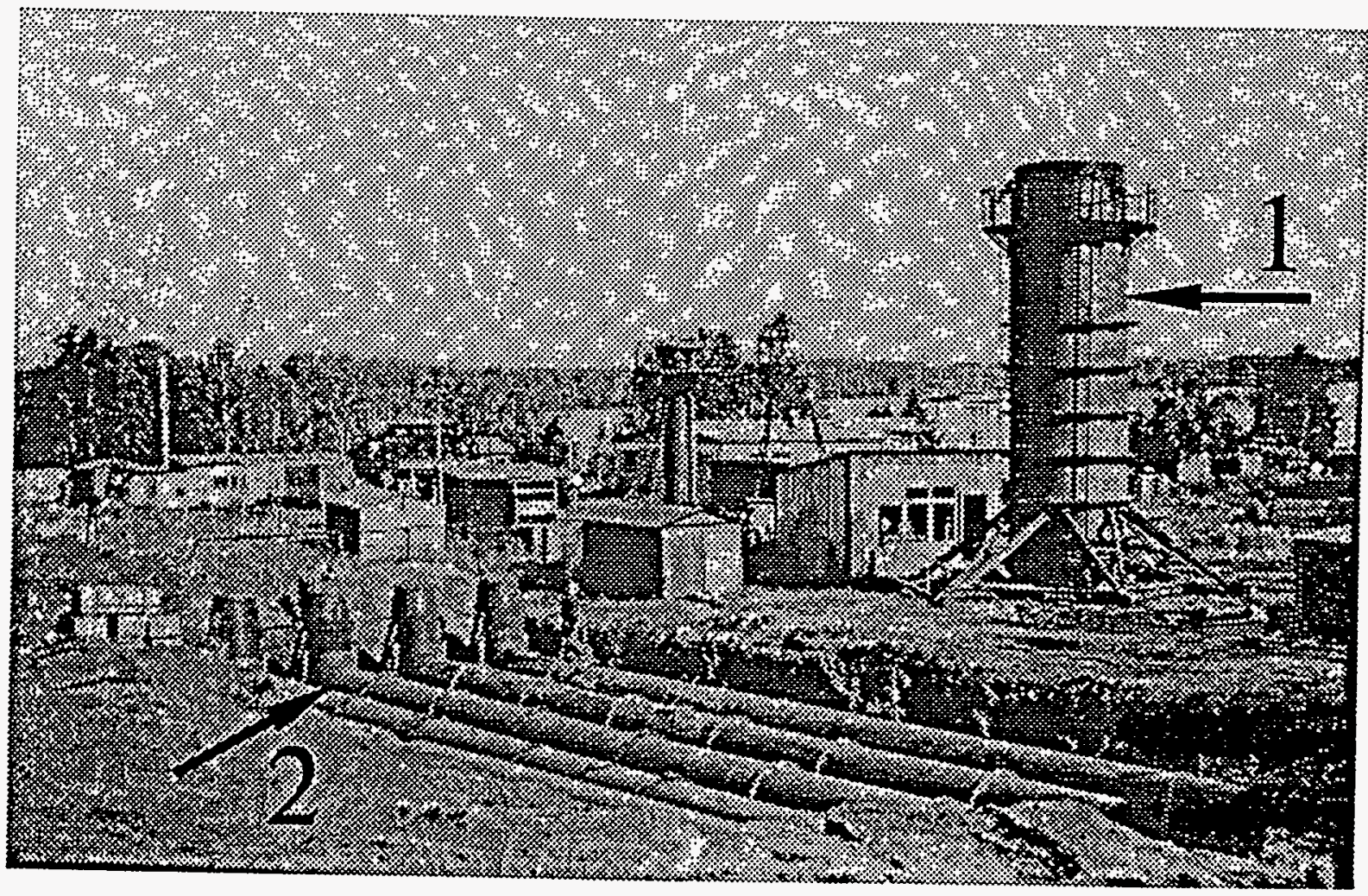

Fig. 10 • 


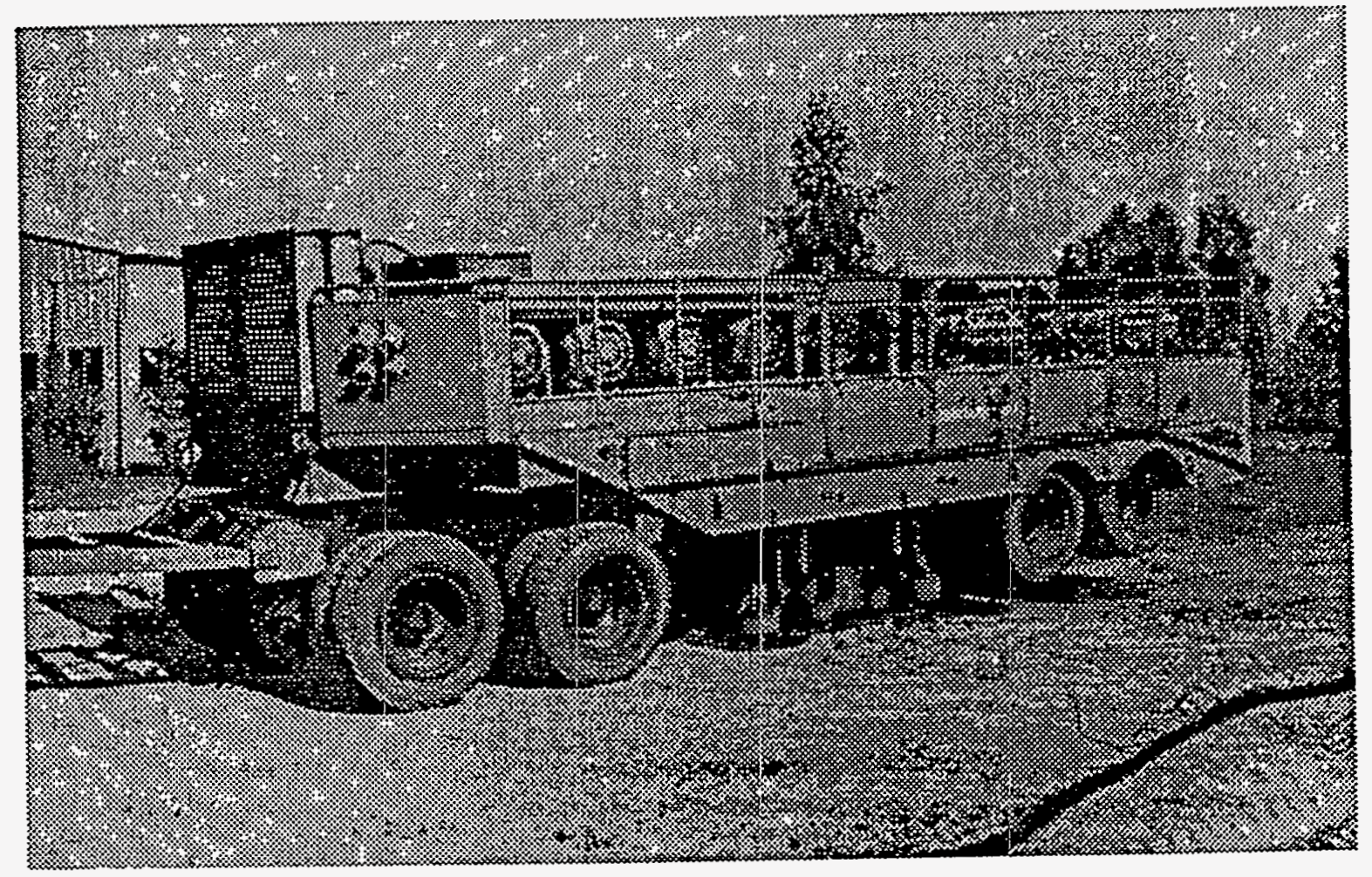

Fig. 11 


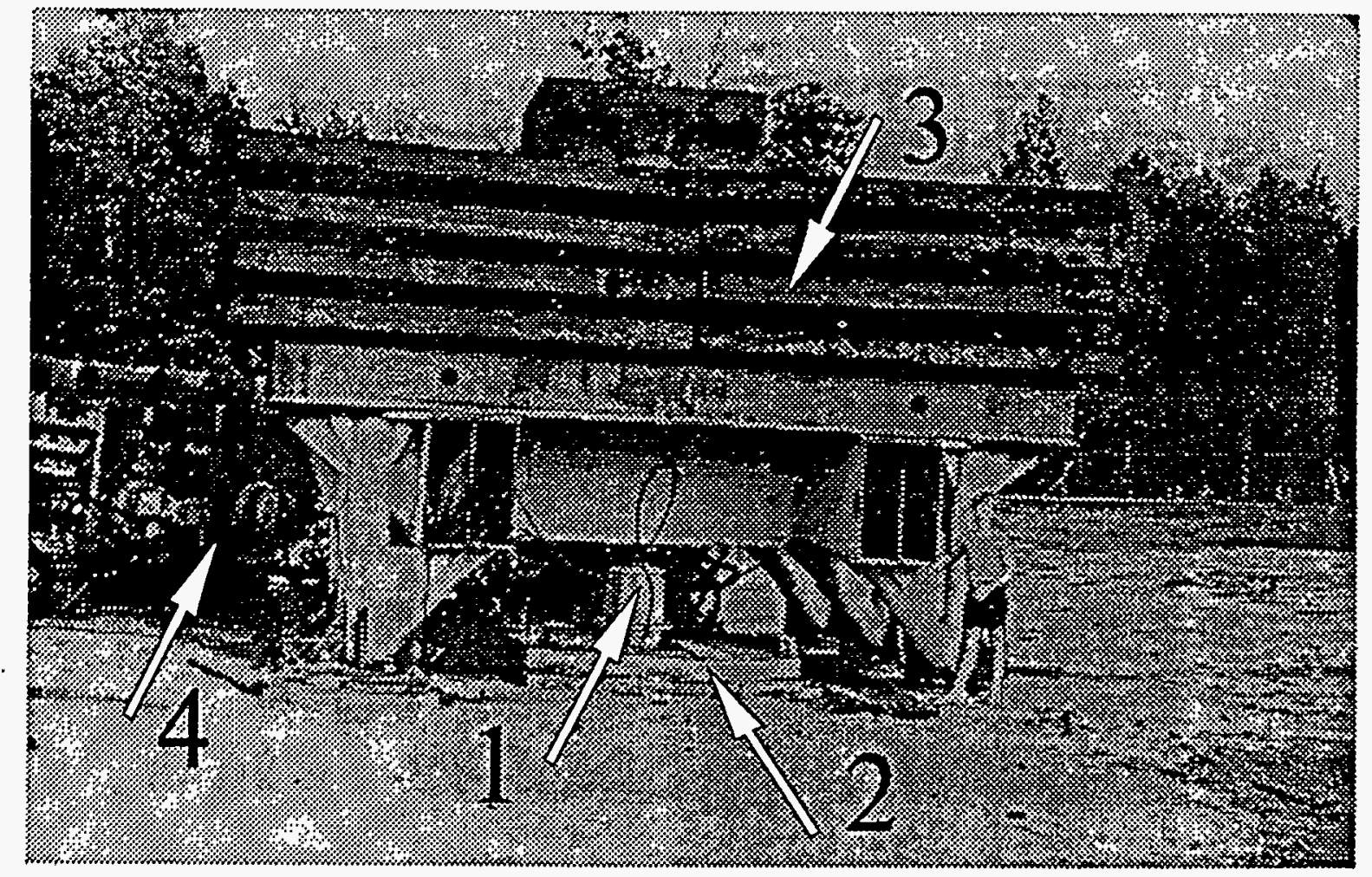

Fig. 12 . 


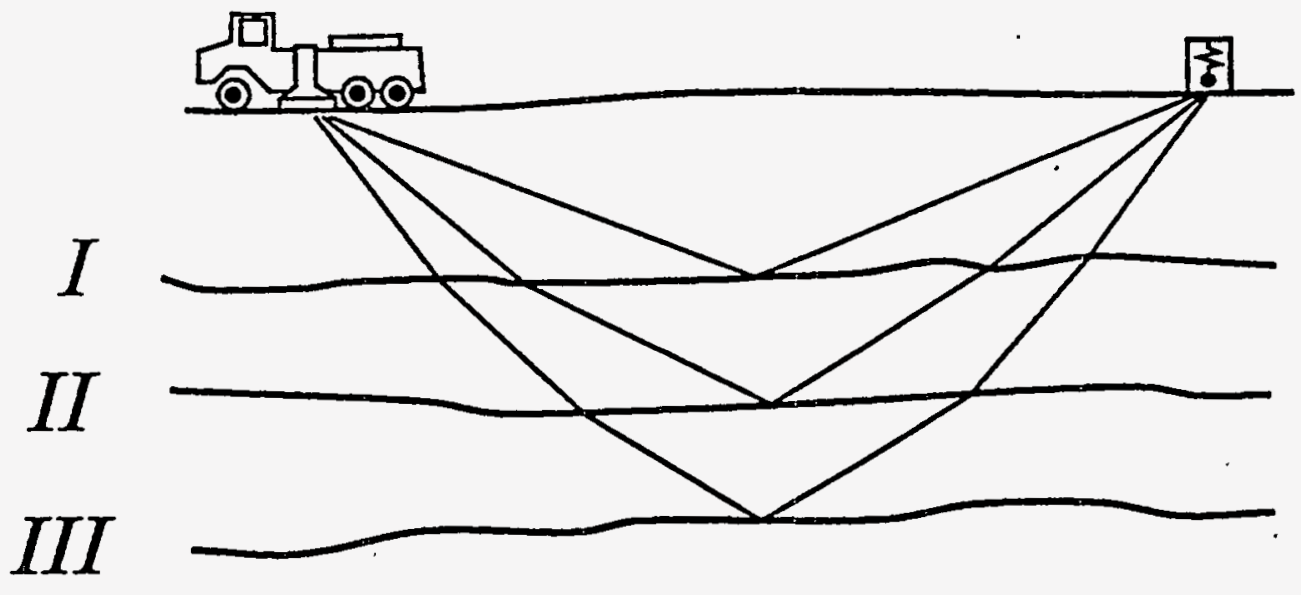

a worron

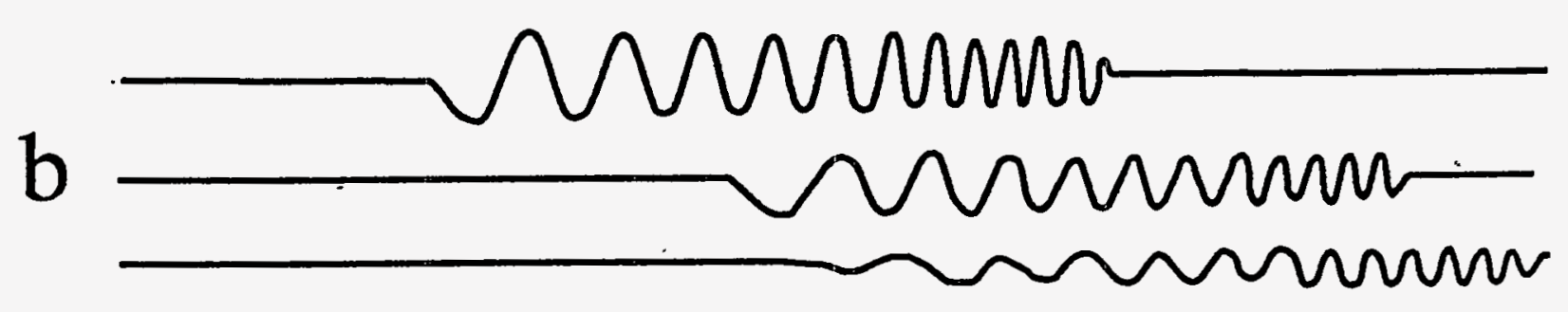

c mowpromprow

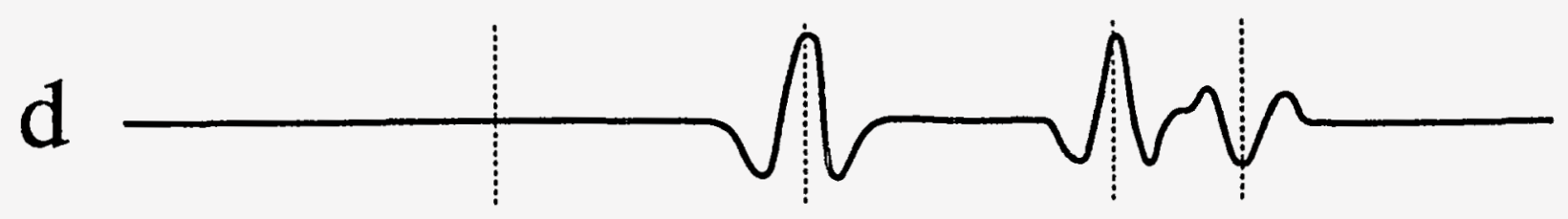
0
$\mathrm{t}_{1}$
$t_{2} t_{3}$

Fig. 13 